\title{
MIMO PI Controllers for LTI Systems with Multiple Time Delays Based on ILMIs and Sensitivity Functions
}

\author{
Wajdi Belhaj and Olfa Boubaker \\ National Institute of Applied Sciences and Technology (INSAT), Centre Urbain Nord, BP 676, 1080 Tunis Cedex, Tunisia \\ Correspondence should be addressed to Olfa Boubaker; olfa.boubaker@insat.rnu.tn
}

Received 12 August 2016; Revised 9 November 2016; Accepted 5 December 2016; Published 20 February 2017

Academic Editor: Tamas Kalmar-Nagy

Copyright (c) 2017 Wajdi Belhaj and Olfa Boubaker. This is an open access article distributed under the Creative Commons Attribution License, which permits unrestricted use, distribution, and reproduction in any medium, provided the original work is properly cited.

\begin{abstract}
In this paper, a MIMO PI design procedure is proposed for linear time invariant (LTI) systems with multiple time delays. The controller tuning is established in two stages and guarantees performances for set-point changes, disturbance variations, and parametric uncertainties. In the first stage, an iterative linear matrix inequality (ILMI) approach is extended to design PI controllers for systems with multiple time delays without performance guarantee, a priori. The second stage is devoted to improve the closedloop performances by minimizing sensitivity functions. Simulations results carried out on the unstable distillation column, the stable industrial scale polymerization (ISP) reactor, and the non-minimum phase 4-tank benchmark prove the efficiency of the proposed approach. A comparative analysis with the conventional internal model control (IMC) approach, a multiloop IMC-PI approach, and a previous ILMI PID approach proves the superiority of the proposed approach compared to the related ones.
\end{abstract}

\section{Introduction}

PID controllers have been at the heart of control engineering practice for several decades $[1,2]$. They are widely used in industrial applications as no other controllers match simple control structure, fewer tuning parameters, and robustness against uncertainties. However, until now, a high percentage of PID control systems seem to be badly tuned and many difficulties occur essentially when the multi-input multioutput systems are considered [3-5]. One major reason may be explained by coupling interactions between the different loops and mainly the negligence of uncertain and immeasurable dead times. Tuning multiloop PID controllers for LTI systems with multiple time delays [6-9] is then considered until now as a challenging problem in control theory. In this framework, the internal model control (IMC) method is considered as the most conventional and effective approach for PID controller design while taking into account time delays [10-12]. The design of MIMO IMC-PID controllers is based on a series of SISO controllers using IMC interaction measures between the different loops. This method becomes very hard when the number of inputs/outputs increases. Even more, its implementation may fail when the interaction measures between the different loops are so high. The last difficulty represents the main disadvantage of this method and an alternative solution was proposed by Vu and Lee [13] to solve such a problem. Unfortunately, this result remains applicable only when the first-order MIMO systems are considered.

On the other hand, iterative linear matrix inequalities (ILMIs) are known to be powerful tools to solve multivariable control problems. Particularly, ILMI approaches were already used to design PID controllers for LTI systems without delays [14-18]. The basic idea was based on transforming the PID controller into an equivalent static output feedback (SOF) stabilization one by augmenting, using some new state variables, the dimension of the controlled system. Unfortunately, such controllers are known by their bad performances compared to those designed via IMC approaches (when applicable).

As Loop Shaping (LS) techniques $[19,20]$ are well known for their abilities to improve the closed-loop system performances by minimizing the signal transmission from 
load disturbances and measurement noise to input and output process or in terms of requirements on the sensitivity functions and/or complementary sensitivity functions [2123], this paper suggests using this concept for improving the MIMO PI controller performances computed via ILMIs. The proposed approach overcomes the problems introduced by the well known IMC method when the fully cross-coupled multivariable systems are considered. Its implementation requires two steps: in the first step, the ILMI method proposed by Zheng et al. [14], appropriate for systems without delays, is extended for the design of PI controller for multiple time delay systems. As such approach generally gives bad performances, a second stage is then launched in order to improve the performances of the closed-loop system by shaping the already designed PI controller by minimizing the sensitivity function of the system.

To illustrate the effectiveness and the performances of the proposed approach, three examples of multiple time delay systems including unstable, stable, and non-minimum systems are considered. A comparative analysis with related approaches is also given to prove the superiority of the proposed approach.

The paper is organized as follows: The problem formulation is stated in Section 2. Model reduction of the MIMO system with multiple time delays is detailed in Section 3. Section 4 is devoted to the main results. Section 5 shows the validity of the proposed approach where a comparative study with related approaches using typical examples for set-point tracking, disturbance rejection, and parametric uncertainties scenarios is considered.

\section{Problem Statement}

Consider a nominal multivariable LTI system with multiple time delays described by

$$
\begin{aligned}
\dot{x}(t)= & A_{0} x(t)+A_{1} x\left(t-\tau_{1}\right)+B_{0} u\left(t-\tau_{2}\right) \\
& +B_{1} u\left(t-\tau_{3}\right), \\
y(t)= & C x(t),
\end{aligned}
$$

where $x(t) \in \mathfrak{R}^{n}, u(t) \in \mathfrak{R}^{m}$, and $y(t) \in \mathfrak{R}^{p}$ are the state vector, the control vector, and the output vector, respectively. $A_{0} \in \mathfrak{R}^{n \times n}, A_{1} \in \mathfrak{R}^{n \times n}, B_{0} \in \mathfrak{R}^{n \times m}, B_{1} \in \mathfrak{R}^{n \times m}$, and $C \in \mathfrak{R}^{p \times n}$ are known constant matrices. $\tau_{1}, \tau_{2}$, and $\tau_{3}$ are time delays.

The objective is to design a finite dimensional PI controller described by

$$
u(t)=F_{1} e(t)+F_{2} \int_{0}^{t} e(t) d t
$$

where $e(t)=r(t)-y(t), r(t) \in \mathfrak{R}^{p}$, is the set-point vector and $F_{1}, F_{2} \in \mathfrak{R}^{m \times p}$ are proportional and time integral gain matrices, respectively, that stabilize the system (1) to the setpoint vector.

Let $G(s) \in \mathfrak{R}^{p \times m}$ be the general transfer matrix of the delayed system (1), computed as described in [24], and $K(s)=$
$\left[K_{i j}(s)\right] \in \mathfrak{R}^{m \times p}$ the transfer matrix of the PI controller given by

$$
K_{i j}(s)=F_{1_{i j}}+\frac{F_{2_{i j}}}{s},
$$

where $K_{i j}(s)$ is the $i j$ th element of the transfer matrix $K(s)$, $F_{1_{i j}}$ is the proportional gain of the $i j$ th element of $K(s)$, and $F_{2_{i j}}$ is the integral gain of the $i j$ th element of $K(s)$,

For such PI controller there are $2 \times m \times p$ parameters to be tuned for a plant with $m$ inputs and $p$ outputs.

The last control problem is very complex since system (1) is a MIMO infinite dimensional system. To be relaxed, the control problem will be organized in two subproblems.

2.1. Subproblem 1: Design a Finite Dimensional PI Controller for Just SOF Stabilization. In this stage, the infinite dimensional system (1) will be reduced to the finite dimensional system (4)-(5) whereas the PI controller (2) will be transformed into the SOF controller (6) described, respectively, by

$$
\begin{aligned}
& \dot{\tilde{z}}=\widetilde{A} \widetilde{z}+\widetilde{B} u, \\
& \tilde{y}=\widetilde{C} \widetilde{z}, \\
& u=\widetilde{F} \widetilde{y},
\end{aligned}
$$

where $\widetilde{z}(t) \in \mathfrak{R}^{n^{\prime}}, u(t) \in \mathfrak{R}^{m}$, and $\tilde{y}(t) \in \mathfrak{R}^{p^{\prime}}$ are the sate vector, the control vector, and the output vector of the approximated system, respectively. $\widetilde{A} \in \mathfrak{R}^{n^{\prime} \times n^{\prime}}, \widetilde{B} \in \mathfrak{R}^{n^{\prime} \times m}$, and $\widetilde{C} \in \Re^{p^{\prime} \times n^{\prime}}$ are matrices related to the approximated system to be computed using the approximation method and the SOF transformation and $\widetilde{F} \in \mathfrak{R}^{m \times p^{\prime}}$ and is the SOF feedback gain matrix, to be designed under the following assumptions.

Assumption 1. The set-point vector $r(t)$ in (2) is assumed to be null $(r(t)=0)$.

Assumption 2. $\tau_{1}, \tau_{2}$, and $\tau_{3}$ are assumed to be uncertain but constant delays.

Assumption 3. The PI controller (2) is well-posed.

Assumption 4. The finite dimensional closed-loop dynamics $\dot{\vec{z}}=(\widetilde{A}+\widetilde{B} \widetilde{F} \widetilde{C}) \widetilde{z}$ with a realization $(\widetilde{A}, \widetilde{B}, \widetilde{C})$ is stabilizable via SOF controller.

To this end, the PI design procedure is proposed in Section 4.1.

2.2. Subproblem 2: Set-Point Stabilization and Output Disturbance Attenuation by Minimizing Sensitivity Functions. The objective of the subproblem 2 is to design a shaped controller described by

$$
K_{\mathrm{sh}}(s)=V_{1}(s) K(s) V_{2}(s)
$$

that improves the closed-loop response considering a shaped system described by

$$
G_{\mathrm{sh}}(s)=V_{1}(s) G(s) V_{2}(s),
$$




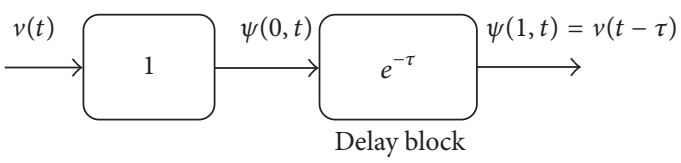

FIgURE 1: Modeling a delayed variable via a distributed parameter system.

where $V_{1}(s) \in \mathfrak{R}^{p \times p}$ and $V_{2}(s) \in \mathfrak{R}^{m \times m}$ are a preand postcompensators to be chosen in order to satisfy, in closed-loop, performance specifications such as set-point stabilization and load disturbance rejection.

The most crucial part of the design procedure is to find the appropriate weighting matrices $V_{1}(s)$ and $V_{2}(s)$. Note that the shape of the weights is determined by the closed-loop design specifications. Once the desired loop shape is achieved, the final controller $K_{\text {fin }}(s)$ to be applied to the nominal transfer matrix $G(s)$ is then constructed. To this end, a Loop Shaping design procedure is proposed in Section 4.2.

\section{Model Reduction of the MIMO System with Multiple Time Delays}

Each delayed variable can be modeled as a distributed parameter system described by a partial differential equation as follows [25]:

$$
\frac{\partial \psi(z, t)}{\partial t}=-\frac{1}{\tau} \frac{\partial \psi(z, t)}{\partial z}
$$

with the boundary condition

$$
v(t)=\psi(0, t)
$$

and the output equations:

$$
v(t-\tau)=\psi(1, t)
$$

where $t$ and $z$ are time and pseudospace variables, respectively. As shown by Figure 1, $v(t), \psi(z, t)$, and $v(t-\tau)$ are the input, the state variable, and the output of the delay block, respectively. $\tau$ is a constant time delay.
For numerical simulation or control design purposes, an infinite dimensional system is generally reduced to a finite dimensional system by using an approximation method. Within the framework of weighted residuals methods, the orthogonal collocation method is applied in this paper to approximate the partial differential equations described by relation (9) augmented by boundary conditions (10)-(11) for its simplicity since it avoids integration [26].

The principle of the orthogonal collocation method is to search a finite dimensional approximation for the distributed parameter variable $\psi(z, t)$ in the following form [27]:

$$
\psi^{*}(z, t)=\sum_{i=0}^{N} c_{i}(t) L_{i}^{N}(z)
$$

where $\psi^{*}$ denotes the approximation of $\psi(z, t) ; N$ is the order reduction; $c_{i}(t)$ are unknown time-varying coefficients chosen such that the approximated solution is the exact one at the collocation points such that

$$
c_{i}(t)=\psi^{*}\left(z_{i}, t\right)=\left.\psi(z, t)\right|_{z=z_{i}}, \quad \forall i \in\{0, \ldots, N\}
$$

and $L_{i}^{N}(z)$ are the $N$ th order Lagrange interpolation polynomials; that is:

$$
L_{i}^{(N)}(z):=\prod_{\substack{j=0 \\ \neq i}}^{N} \frac{z-z_{j}}{z_{j}-z_{i}}
$$

where $z_{0}, z_{1}, \ldots, z_{N} \in[0,1]$ are the collocation points of the method. In this paper, the internal collocation points are considered as the zeros of the $(N+2)$ th order Jacobi polynomial defined for $i=1, \ldots, N+2$ by Lefèvre et al. [27] as follows:

$$
p_{N}^{(p, q)}=\left(z-g_{N}^{(p, q)}\right) p_{N-1}^{(p, q)}-h_{N}^{(p, q)} p_{N-2}^{(p, q)}
$$

with $p_{0}^{(p, q)}=1$ and where coefficients $h_{N}^{(p, q)}$ and $g_{N}^{(p, q)}$ are defined as follows:

$$
\begin{aligned}
& h_{N}^{(p, q)}:= \begin{cases}\frac{(N-1)(N+p-1)(N+q-1)(N+p+q-1)}{(2 N+p+q-1)(2 N+p+q-2)^{2}(2 N+p+q-3)}, & \text { if } N>2, \\
\frac{(p+1)(q+1)}{(p+q+2)^{2}(p+q+3)}, & \text { if } N=2,\end{cases} \\
& g_{N}^{(p, q)}:= \begin{cases}0, & \text { otherwise, } \\
\frac{1}{2}\left(1-\frac{p^{2}-q^{2}}{(2 N+p+q-1)^{2}-1}\right), & \text { if } N>1, \\
\frac{q+1}{p+q+2}, & \text { if } N=1,\end{cases}
\end{aligned}
$$

where $p$ and $q$ are two constant parameters affecting the position of the collocation points.
By applying delay variable approximation on each delayed variable of the vectors $x\left(t-\tau_{1}\right), u\left(t-\tau_{2}\right)$, and $u\left(t-\tau_{3}\right)$, the 
following $3(N+1)$ finite dimensional equations can be then obtained from the partial differential equation (9), [28]:

$$
\begin{aligned}
& \dot{\psi}_{1}(t)=-\frac{1}{\tau_{1}} \bar{A}_{1} \psi_{1}(t)+\frac{1}{\tau_{1}} \bar{B}_{1} x(t), \\
& \dot{\psi}_{2}(t)=-\frac{1}{\tau_{2}} \bar{A}_{2} \psi_{2}(t)+\frac{1}{\tau_{2}} \bar{B}_{2} u(t), \\
& \dot{\psi}_{3}(t)=-\frac{1}{\tau_{3}} \bar{A}_{3} \psi_{3}(t)+\frac{1}{\tau_{3}} \bar{B}_{3} u(t),
\end{aligned}
$$

augmented by the following outputs:

$$
\begin{aligned}
& x\left(t-\tau_{1}\right)=\psi_{1}(1, t)=\bar{C}_{1} \psi_{1}(t), \\
& u\left(t-\tau_{2}\right)=\psi_{2}(1, t)=\bar{C}_{2} \psi_{2}(t), \\
& u\left(t-\tau_{3}\right)=\psi_{3}(1, t)=\bar{C}_{3} \psi_{3}(t),
\end{aligned}
$$

where for $k=1,2,3, \bar{A}_{i}, \bar{B}_{i}, \bar{C}_{i}$ are computed as given in [28].

Let consider Cauchy's formula for the interpolation error defined by Lefèvre et al. [27] as follows:

$$
e_{N}(z, t)=\psi(z, t)-\psi^{*}(z, t)
$$

and assume that the unknown solution $\psi(z, t)$ is sufficiently continuously differentiable; we have then

$$
e_{N}(z, t)=w(z) \frac{\psi(z)^{(N+1)}(\eta(z), t)}{(N+1) !},
$$

where $w(z):=\prod_{j=0}^{N}\left(z-z_{j}\right)$ and $\eta(z) \in[-1,+1]$.

Hence, we try to choose the interior collocation points $z_{1}, \ldots, z_{N-1}$ that minimize the interpolation error (19). Without any a priori knowledge on the behavior of the exact solution, this problem reduces to finding $z_{1}, \ldots, z_{N-1}$ such that $\psi(z)$ is minimal.

By considering the case study of the Chebyshev polynomials belonging to the family of Jacobi polynomials, and, corresponding to the values of the parameters $p=q=-1 / 2$, the corresponding minimal norm is given by Lefèvre et al. [27] as follows:

$$
\left\|e_{N}\right\|_{\infty} \leq \frac{\left\|\psi(z)^{N+1}(\eta(z), t)\right\|_{\infty}}{(N+1) ! 2^{N-2}} .
$$

Through the result (21), we demonstrate that the interpolation error for a variable delay approximation is always bounded for the parameters $p=q=-1 / 2$.

\section{Main Results}

4.1. PI Controller Design via ILMIs. In the following, the SOF transformation of the PI controller of the delayed system (1) will be detailed. Using (18), the system (1) can be written as follows:

$$
\begin{aligned}
\dot{x}(t)= & A_{0} x(t)+A_{1} \bar{C}_{1} \psi_{1}(t)+B_{0} \bar{C}_{2} \psi_{2}(t) \\
& +B_{1} \bar{C}_{3} \psi_{3}(t), \\
y(t)= & C x(t) .
\end{aligned}
$$

Let now

$$
\widetilde{z}^{T}=\left[\begin{array}{ll}
\widetilde{z}_{1}^{T} & \widetilde{z}_{2}^{T}
\end{array}\right]
$$

where:

$$
\begin{aligned}
\widetilde{z}_{1} & =\left[\begin{array}{llll}
x(t) & \psi_{1}(t) & \psi_{2}(t) & \psi_{3}(t)
\end{array}\right] \in \mathfrak{R}^{4 n}, \\
\widetilde{z}_{2}(t) & =\int_{0}^{t} y(t) d t
\end{aligned}
$$

and let:

$$
\widetilde{y}=\left[\begin{array}{ll}
\widetilde{y}_{1} & \widetilde{y}_{2}
\end{array}\right]^{T}=\widetilde{C} \widetilde{z},
$$

where:

$$
\begin{aligned}
& \tilde{y}_{1}=y=C x=\left[\begin{array}{lllll}
C & 0 & 0 & 0 & 0
\end{array}\right] \widetilde{z}, \\
& \widetilde{y}_{2}=\int_{0}^{t} y(t) d t=\left[\begin{array}{lllll}
0 & 0 & 0 & 0 & I
\end{array}\right] \widetilde{z} .
\end{aligned}
$$

The state space of a new augmented system controlled via an SOF controller is then deduced as follows:

$$
\begin{aligned}
\dot{\tilde{z}} & =\widetilde{A} \widetilde{z}+\widetilde{B} u, \\
\tilde{y} & =\widetilde{C} \widetilde{z}, \\
u & =\widetilde{F} \widetilde{y},
\end{aligned}
$$

where:

$$
\begin{aligned}
& \widetilde{A}=\left(\begin{array}{ccccc}
A_{0} & A_{1} \bar{C}_{1} & B_{0} \bar{C}_{2} & B_{1} \bar{C}_{3} & 0 \\
\frac{1}{\tau_{1}} \bar{B}_{1} & -\frac{1}{\tau_{1}} \bar{A}_{1} & 0 & 0 & 0 \\
0 & 0 & -\frac{1}{\tau_{2}} \bar{A}_{2} & 0 & 0 \\
0 & 0 & 0 & -\frac{1}{\tau_{3}} \bar{A}_{3} & 0 \\
C & 0 & 0 & 0 & 0
\end{array}\right) \\
& \in \Re^{n^{\prime} \times n^{\prime}} \\
& \widetilde{B}=\left(\begin{array}{c}
0 \\
0 \\
\frac{1}{\tau_{2}} \bar{B}_{2} \\
\frac{1}{\tau_{3}} \bar{B}_{3} \\
0
\end{array}\right) \in \Re^{n^{\prime} \times m} \\
& \widetilde{C}=\left[\begin{array}{ll}
\widetilde{C}_{1}^{T} & \widetilde{C}_{2}{ }^{T}
\end{array}\right]^{T} \in \mathfrak{R}^{p^{\prime} \times n^{\prime}}, \\
& \widetilde{C}_{1}=\left[\begin{array}{lllll}
C & 0 & 0 & 0 & 0
\end{array}\right] \in \mathfrak{R}^{p \times n^{\prime}}, \\
& \widetilde{C}_{2}=\left[\begin{array}{lllll}
0 & 0 & 0 & 0 & I_{p \times p}
\end{array}\right] \in \mathfrak{R}^{p \times n^{\prime}} .
\end{aligned}
$$

Taking into account (27), the control law (2) under Assumption 1 can be written as follows:

$$
u=-\left(F_{1} \tilde{y}_{1}+F_{2} \tilde{y}_{2}\right) \text {. }
$$


On the other hand, we have from (27) the following:

$$
u=-\left(\widetilde{F}_{1} \widetilde{y}_{1}+\widetilde{F}_{2} \widetilde{y}_{2}\right)=\widetilde{F} \widetilde{y}
$$

We can deduce that once the matrix $\widetilde{F}=\left[\begin{array}{ll}-\widetilde{F}_{1} & -\widetilde{F}_{2}\end{array}\right] \in \mathfrak{R}^{m \times p^{\prime}}$ is designed the closed-loop system (4)-(6) is asymptotically stable. Considering analogy between (29) and (30), the original PI gains can be recovered as follows:

$$
\left[\begin{array}{ll}
F_{1} & F_{2}
\end{array}\right]=\left[\begin{array}{ll}
\widetilde{F}_{1} & \widetilde{F}_{2}
\end{array}\right]
$$

Theorem 5. The multivariable LTI system with multiple time delays (1) is stabilizable via SOF if and only if there exist a constant matrix $\widetilde{F}=\left[\begin{array}{ll}-\widetilde{F}_{1} & -\widetilde{F}_{2}\end{array}\right] \in \mathfrak{R}^{m \times p^{\prime}}$ and a symmetric positive definite matrix $X=X^{T}>0, X \in \Re^{n^{\prime} \times n^{\prime}}$ satisfying the following matrix inequality:

$$
\begin{aligned}
& \widetilde{A}^{T} X+X \widetilde{A}-X \widetilde{B} \widetilde{B}^{T} X+\left(\widetilde{B}^{T} X+\widetilde{F} \widetilde{C}\right)^{T}\left(\widetilde{B}^{T} X+\widetilde{F} \widetilde{C}\right) \\
& \quad<0
\end{aligned}
$$

such that

$$
\begin{aligned}
& F_{1}=\widetilde{F}_{1}, \\
& F_{2}=\widetilde{F}_{2} .
\end{aligned}
$$

Proof.

Sufficiency. Note that

$$
\begin{aligned}
(\widetilde{A}+\widetilde{B} \widetilde{F} \widetilde{C})^{T} X+X(\widetilde{A}+\widetilde{B} \widetilde{F} \widetilde{C}) \\
\leq(\widetilde{A}+\widetilde{B} \widetilde{F} \widetilde{C})^{T} X+X(\widetilde{A}+\widetilde{B} \widetilde{F} \widetilde{C})+\widetilde{C}^{T} \widetilde{F}^{T} \widetilde{F} \widetilde{C} \\
=\widetilde{A}^{T} X+X \widetilde{A}-X \widetilde{B} \widetilde{B}^{T} X \\
\quad+\left(\widetilde{B}^{T} X+\widetilde{F} \widetilde{C}\right)^{T}\left(\widetilde{B}^{T} X+\widetilde{F} \widetilde{C}\right)<0 .
\end{aligned}
$$

From Lyapunov's theory, the closed-loop system $\sum_{c}: \dot{\tilde{z}}=(\widetilde{A}+$ $\widetilde{B} \widetilde{F C}) z$ is then asymptotically stable.

Necessity. Suppose that $\sum_{c}$ is asymptotically stable for some $\widetilde{F}$. Then there exists $X=X^{T}>0$ such that

$$
(\widetilde{A}+\widetilde{B} \widetilde{F} \widetilde{C})^{T} X+X(\widetilde{A}+\widetilde{B} \widetilde{F} \widetilde{C})<0 .
$$

It is easy to find that there exists a scalar $\rho>0$ such that

$$
(\widetilde{A}+\widetilde{B} \widetilde{F} \widetilde{C})^{T} X+X(\widetilde{A}+\widetilde{B} \widetilde{F} \widetilde{C})+\frac{1}{\rho^{2}} \widetilde{C}^{T} \widetilde{F} \widetilde{F} \widetilde{C}<0 ;
$$

that is,

$$
\begin{aligned}
& \widetilde{A}^{T} X+X \widetilde{A}-\rho^{2} X \widetilde{B} \widetilde{B}^{T} X \\
& +\left(\rho \widetilde{B}^{T} X+\frac{1}{\rho} \widetilde{F} \widetilde{C}\right)^{T}\left(\rho \widetilde{B}^{T} X+\frac{1}{\rho} \widetilde{F} \widetilde{C}\right)<0, \\
& \widetilde{A}^{T} X+X \widetilde{A}-\rho^{2} X \widetilde{B} \widetilde{B}^{T} X \\
& +\left(\rho \widetilde{B}^{T} X+\frac{1}{\rho} \widetilde{F} \widetilde{C}\right)^{T}\left(\rho \widetilde{B}^{T} X+\frac{1}{\rho} \widetilde{F} \widetilde{C}\right)<0 .
\end{aligned}
$$

Obviously, (37) is equivalent to

$$
\begin{aligned}
& \rho^{2} \widetilde{A}^{T} X+\rho^{2} X \widetilde{A}-\rho^{4} X \widetilde{B} \widetilde{B}^{T} X \\
& \quad+\left(\rho^{2} \widetilde{B}^{T} X+\widetilde{F} \widetilde{C}\right)^{T}\left(\rho^{2} \widetilde{B}^{T} X+\widetilde{F} \widetilde{C}\right)<0
\end{aligned}
$$

By substituting $\rho^{2} X$ with $X$ in (38), we obtain inequality (32). Condition (33) is already proved in (31).

Due to the term $-X \widetilde{B} \widetilde{B}^{T} X$, (32) cannot be simplified to an LMI. Similarly to SOF control problem described in [1416], an ILMI algorithm can be addressed to solve the Bilinear Matrix Inequality (BMI) in (32). To accommodate the $-X \widetilde{B} \widetilde{B}^{T} X$ term, an additional design variable $Y$ is introduced. Because $(Y-X)^{T} \widetilde{B} \widetilde{B}^{T}(Y-X) \geq 0$ for any $Y>0$ and $X$ for the same dimension, we obtain

$$
Y^{T} \widetilde{B} \widetilde{B}^{T} X+X^{T} \widetilde{B} \widetilde{B}^{T} Y-Y^{T} \widetilde{B} \widetilde{B}^{T} Y \leq X^{T} \widetilde{B} \widetilde{B}^{T} X
$$

By combining (32) and (39), a sufficient condition for the existence of SOF gain matrix $\widetilde{F}$ is obtained such that

$$
\begin{gathered}
\widetilde{A}^{T} X+X \widetilde{A}-Y \widetilde{B} \widetilde{B}^{T} X-X \widetilde{B} \widetilde{B}^{T} Y+Y \widetilde{B} \widetilde{B}^{T} Y \\
+\left(\widetilde{B}^{T} X+\widetilde{F} \widetilde{C}\right)^{T}\left(\widetilde{B}^{T} X+\widetilde{F} \widetilde{C}\right)<0 .
\end{gathered}
$$

Considering the $\alpha / 2$ stabilizability via SOF concept [29], if the matrix inequality (40) has a solution $(X>0, \widetilde{F})$, then there exist a real number $\alpha \geq 0$ and a fixed matrix $Y>0$ such that

$$
\begin{aligned}
\widetilde{A}^{T} X & +X \widetilde{A}-Y \widetilde{B} \widetilde{B}^{T} X-X^{T} \widetilde{B} \widetilde{B}^{T} Y+Y^{T} \widetilde{B} \widetilde{B}^{T} Y \\
& +\left(\widetilde{B}^{T} X+\widetilde{F} \widetilde{C}\right)^{T}\left(\widetilde{B}^{T} X+\widetilde{F} \widetilde{C}\right)-\alpha X<0 .
\end{aligned}
$$

Based on the idea that all eigenvalues of $\widetilde{A}+\widetilde{B} \widetilde{F} \widetilde{C}$ are shifted progressively towards the left-half-plane through the reduction of $\alpha$, we may close in on the feasibility of (32) [29]. Using Schur complement, inequality (41) is equivalent to the following matrix inequality:

$$
\left[\begin{array}{cc}
\widetilde{A}^{T} X+X \widetilde{A}-Y \widetilde{B} \widetilde{B}^{T} X-X^{T} \widetilde{B} \widetilde{B}^{T} Y+Y^{T} \widetilde{B} \widetilde{B}^{T} Y-\alpha X & \left(\widetilde{B}^{T} X+\widetilde{F} \widetilde{C}\right)^{T} \\
\left(\widetilde{B}^{T} X+\widetilde{F} \widetilde{C}\right) & -I
\end{array}\right]<0 .
$$


The previous inequality (42) points to an iterative approach to solve $\widetilde{F}$ and $X>0$; namely, if $Y$ is fixed in (42), then it reduces to an LMI problem in the unknowns $\alpha, \widetilde{F}$ and $X$.

The following is a constructive ILMI algorithm for PI control of LTI MIMO with multiple delays systems, and the explanations are given in Remark 7.

\section{Algorithm 6.}

Step 1. Define the orthogonal collocation method parameters $N, p$, and $q$.

Step 2. Transform the infinite dimensional system (1) to a finite dimensional system (17)-(18) by computing matrices $\bar{A}_{1}, \bar{A}_{2}, \bar{A}_{3}, \bar{B}_{1}, \bar{B}_{2}, \bar{B}_{3}, \bar{C}_{1}, \bar{C}_{2}$, and $\bar{C}_{3}$.

Step 3. Design the SOF transformation to give the system's state space realization $(\widetilde{A}, \widetilde{B}, \widetilde{C})$. If it does proceed to Step 4 .

Step 4. Set $i=1$ and choose $Y_{1}=I_{n^{\prime}}+\varepsilon_{1}$ where $\varepsilon_{1} \geq 0$.

Step 5. Solve the following optimization problem for $X_{i}, \widetilde{F}$, and $\alpha_{i}$ :

OP1: Minimize $\alpha_{i}$ subject to the following LMI constraints:

$$
\left[\begin{array}{cc}
\Sigma_{1 i} & \left(\widetilde{B}^{T} X_{i}+\widetilde{F} \widetilde{C}\right)^{T} \\
\widetilde{B}^{T} X_{i}+\widetilde{F} \widetilde{C} & -I
\end{array}\right]<0, \quad X_{i}>0
$$

where $\Sigma_{1 i}=\widetilde{A}^{T} X_{i}+X_{i} \widetilde{A}-Y_{i} \widetilde{B} \widetilde{B}^{T} X_{i}-X_{i} \widetilde{B} \widetilde{B}^{T} Y_{i}+Y_{i} \widetilde{B} \widetilde{B}^{T} Y_{i}-\alpha_{i} X_{i}$ Denote by $\alpha_{i}^{*}$ the minimized value of $\alpha_{i}$.

Step 6. If $\alpha_{i}^{*} \leq 0$, the feedback matrix gains are $F_{1}=\widetilde{F}_{1}$ and $F_{2}=\widetilde{F}_{2}$. Stop. Otherwise, go to Step 7 .

Step 7. Solve the following optimization problem for $X_{i}$ and $\widetilde{F}$.

OP2: Minimize $\operatorname{tr}\left(X_{i}\right)$ subject to LMI constraints (43) with $\alpha_{i}=\alpha_{i}^{*}$, where tr stands for the trace of a square matrix. Denote by $X_{i}^{*}$ the optimal $X_{i}$. The feedback matrix gains are $F_{1}=\widetilde{F}_{1}$ and $F_{2}=\widetilde{F}_{2}$.

Step 8. If $\left\|Y_{i} \widetilde{B}-X_{i}^{*} \widetilde{B}\right\|<\delta$, where $\delta$ is a prescribed tolerance, go to Step 9; otherwise, set $i:=i+1, Y_{i}=X_{i}^{*}$ and go to Step 5.

Step 9. It cannot be decided by this algorithm whether SOF problem is solvable. Stop.

Remark 7. Due to the bad performances generated by initial data selection in ILMI algorithms $[14,15], Y_{1}=I_{n^{\prime}}+\varepsilon_{1}$ is proposed in this paper where $\varepsilon_{1}>0$, yielding to a feasible solution.

4.2. Improving Closed-Loop Performances. In this section, a modified approach of Loop Shaping technique [19] will be introduced to design the multiloop PI controller as

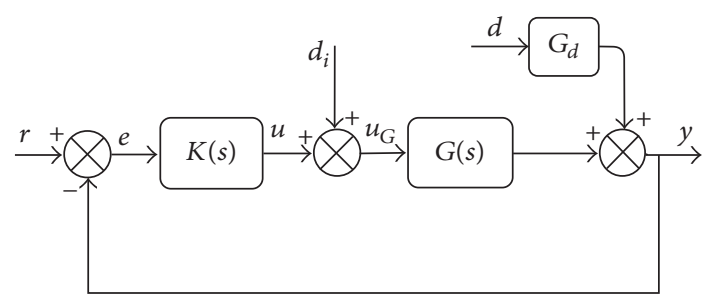

FIGURE 2: Block diagram of the controlled system.

described in subproblem 2 . Figure 2 shows the block diagram of the controlled system where $r, y, u, u_{G}, e, d$ and $d_{i}$ denote the set-point vector, the output vector, the control signal vector, the process control signal vector, the error vector, the output disturbance vector, and the input disturbance vector, respectively.

Let us define the input loop transfer matrix, $L_{i}$, and the output transfer matrix, $L_{o}$, respectively, as follows [20]:

$$
\begin{aligned}
& L_{i}=K G, \\
& L_{o}=G K .
\end{aligned}
$$

The input sensitivity matrix is defined as the transfer matrix from $d_{i}$ to $u_{G}$ such as

$$
\begin{aligned}
S_{i} & =\left(I+L_{i}\right)^{-1}, \\
u_{G} & =S_{i} d_{i}
\end{aligned}
$$

and the output sensitivity matrix is defined as the transfer matrix from $d$ to $y$ such that

$$
\begin{gathered}
S_{o}=\left(I+L_{o}\right)^{-1}, \\
y=S_{o} G_{d} d .
\end{gathered}
$$

The input and output complementary sensitivity matrices are defined as follows:

$$
\begin{aligned}
& T_{i}=I-S_{i}=L_{i}\left(I+L_{i}\right)^{-1}, \\
& T_{o}=I-S_{o}=L_{o}\left(I+L_{o}\right)^{-1} .
\end{aligned}
$$

It is easy to see that the closed-loop system, if it is internally stable, satisfies the following equations:

$$
\begin{gathered}
y=T_{o} r+S_{o} G d_{i}+S_{o} G_{d} d, \\
u=K S_{o} r-K S_{o} G_{d} d-T_{i} d_{i}, \\
e=S_{o}\left(r-G_{d} d\right)-S_{o} G d_{i}, \\
u_{G}=K S_{o} r-K S_{o} G_{d} d+S_{i} d_{i} .
\end{gathered}
$$

Equation (48) shows that the effects of the disturbance $d$ on the plant output $y$ can be made "small" by making the output sensitivity function $S_{o}$ small, as $G_{d}$ is fixed. Similarly, (50) shows the effect of the set-point $r$ to the error $e$ by making $S_{o}$ as small as possible. The notion of smallness for a transfer matrix in a certain range of frequencies can 


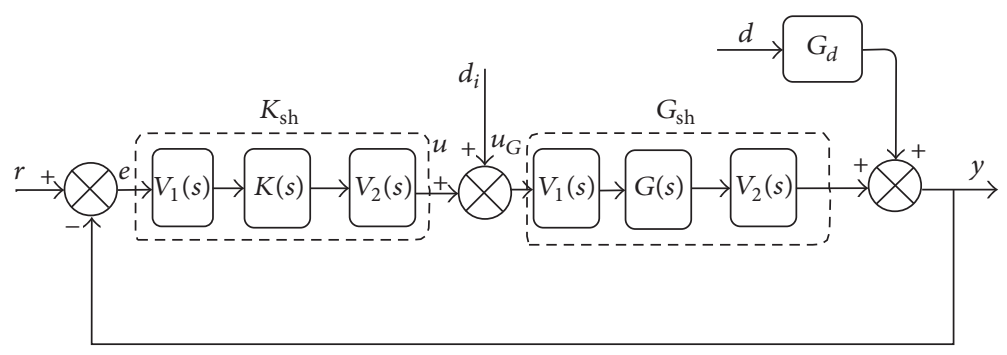

FIGURE 3: Block diagram of a controlled shaped system.

be made explicit using frequency dependent singular values particularly minimizing $\bar{\sigma}\left(S_{o}\right)$ where $\bar{\sigma}$ is the maximum singular value. Similarly to the conventional Loop Shaping design [19, 20], shaping the open-loop nominal system corresponds to shaping the loop gain $G K$ using the preand postcompensators $V_{1}(s)$ and $V_{2}(s)$. The new shaped control system is shown in Figure 3. It is obvious that a well designed control system should meet, at least, the following requirements: (1) stability, (2) set-point tracking, and (3) output disturbance attenuation. Let us then define the input loop transfer matrix, $L_{i s h}$, and the output transfer matrix, $L_{\text {osh }}$, as

$$
\begin{aligned}
& L_{i s h}=K_{\mathrm{sh}} G_{\mathrm{sh}}, \\
& L_{\text {osh }}=G_{\mathrm{sh}} K_{\mathrm{sh}} .
\end{aligned}
$$

The input sensitivity matrix is defined as the transfer matrix from $d_{i}$ to $u_{G}$ as follows:

$$
\begin{aligned}
S_{i s h} & =\left(I+L_{i s h}\right)^{-1}, \\
u_{G} & =S_{i s h} d_{i}
\end{aligned}
$$

and the output sensitivity matrix is defined as the transfer matrix from $d$ to $y$ as follows:

$$
\begin{aligned}
S_{o s h} & =\left(I+L_{o s h}\right)^{-1}, \\
y & =S_{o s h} G_{d} d .
\end{aligned}
$$

The input and output complementary sensitivity matrices are defined as

$$
\begin{aligned}
& T_{i s h}=I-S_{i s h}=L_{i s h}\left(I+L_{i s h}\right)^{-1}, \\
& T_{o s h}=I-S_{o s h}=L_{o s h}\left(I+L_{o s h}\right)^{-1} .
\end{aligned}
$$

It is easy to see that the closed-loop system, if it is internally stable, satisfies the following equations:

$$
\begin{gathered}
y=T_{o s h} r+S_{o s h} G_{\mathrm{sh}} d_{i}+S_{o s h} G_{d} d, \\
u=K_{\mathrm{sh}} S_{o s h} r-K_{\mathrm{sh}} S_{o s h} G_{d} d-T_{i s h} d_{i}, \\
e=S_{o s h}\left(r-G_{d} d\right)-S_{o s h} G_{\mathrm{sh}} d_{i}, \\
u_{G}=K_{\mathrm{sh}} S_{o s h} r-K_{\mathrm{sh}} S_{o s h} G_{d} d+S_{i s h} d_{i} .
\end{gathered}
$$

As $G_{d}$ is fixed, from (56) and (58), good set-point tracking and output disturbance attenuation would require the maximum singular value of the output sensitivity matrix of the shaped system $\bar{\sigma}\left(S_{\text {osh }}\right)$ be made small such as

$$
\begin{aligned}
\bar{\sigma}\left(S_{\text {osh }}\right) & =\bar{\sigma}\left(\left(I+G_{\mathrm{sh}} K_{\mathrm{sh}}\right)^{-1}\right)=\frac{1}{\underline{\sigma}\left(I+G_{\mathrm{sh}} K_{\mathrm{sh}}\right)} \\
& \leq \frac{1}{\underline{\sigma}\left(G_{\mathrm{sh}} K_{\mathrm{sh}}\right)} .
\end{aligned}
$$

It should be indicated that improving the closed-loop shaped system performances over those of the nominal system would require $\bar{\sigma}\left(S_{o s h}\right)$ be made smaller than $\bar{\sigma}\left(S_{o}\right)$, particularly in the low frequency range where $d$ is usually significant. As $G(s)$ and $K(s)$ are fixed by subproblem $1, V_{1}(s)$ and $V_{2}(s)$ play a key role in the Loop Shaping design procedure. Thus, synthesis of the shaped controller $K_{\text {sh }}$ is reduced to choose an appropriate $V_{1}(s)$ and $V_{2}(s)$ in order to guarantee closed-loop performances, under the following:

$$
\min \bar{\sigma}\left(S_{o s h}\right)
$$

such that

$$
\bar{\sigma}\left(S_{o s h}\right)<\bar{\sigma}\left(S_{o}\right)
$$

Figure 4 synthesizes the Loop Shaping design procedure proposed in this paper where the proposed Loop Shaping design procedure is stated below:

\section{Algorithm 8.}

Step 1. Consider the PI controller designed via Algorithm 6. Assume that the closed-loop system performances are not well performed and define the control objectives for the desired closed-loop system responses (good disturbance rejection, steady state error minimization).

Step 2. Choose a precompensator $V_{1}(s)$ and a postcompensator $V_{2}(s)$ such that the singular values of the nominal plant $G(s)$ are shaped to give a desired open-loop shape (high low frequency gain and low high frequency gain).

Step 3. For the shaped plant $G_{\text {sh }}(s)$, if the control objectives and constraint (62) are satisfied then go to Step 4. Else adjust $V_{1}(s)$ and $V_{2}(s)$ and go to Step 2. 


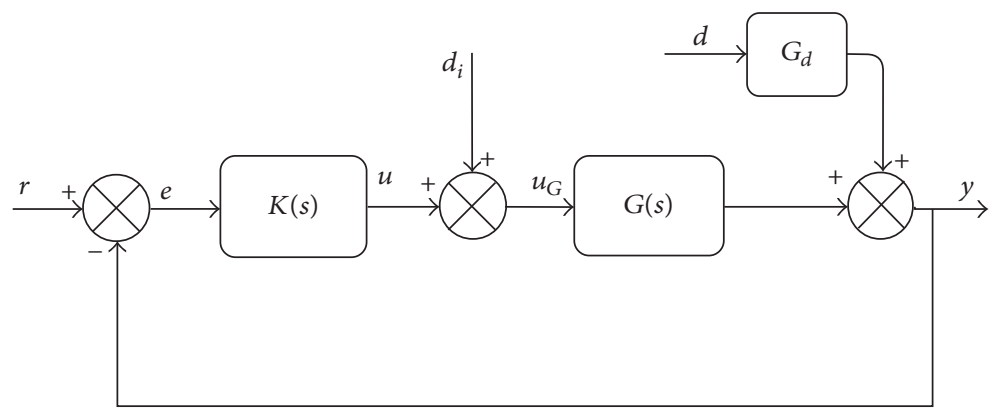

(a)

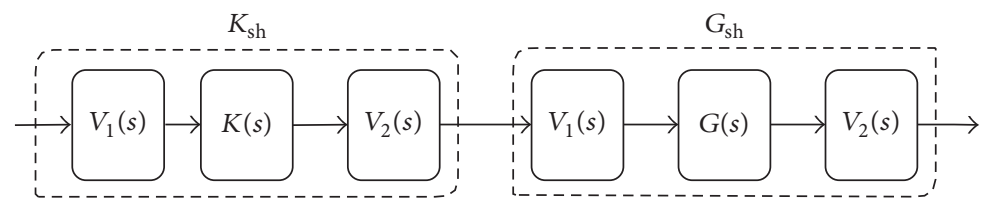

(b)

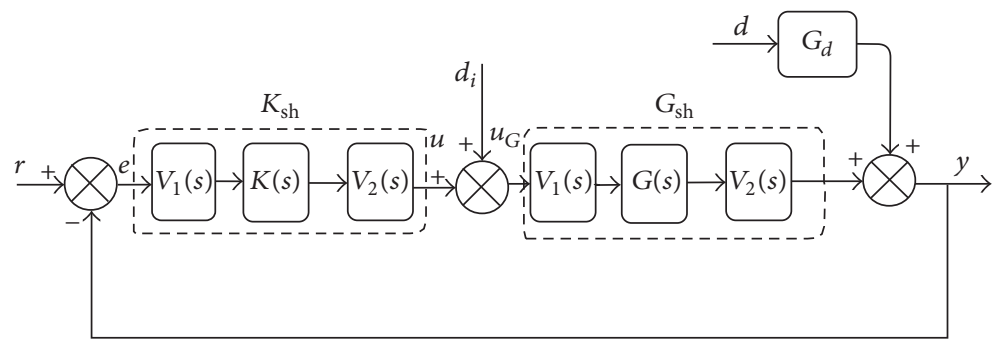

(c)

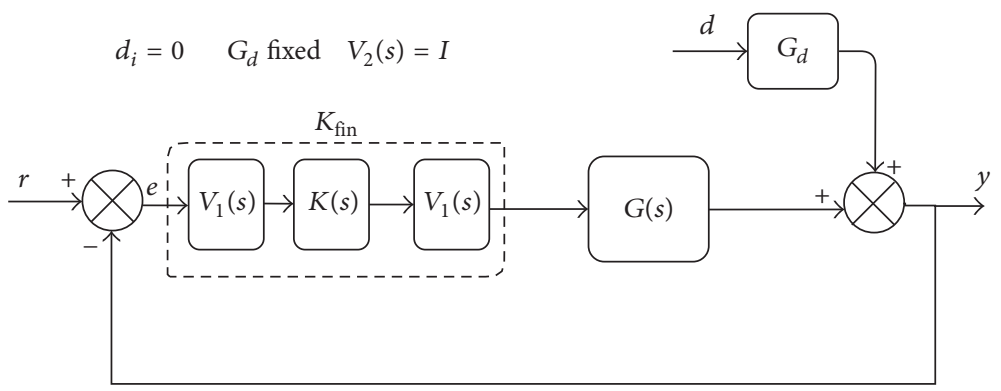

(d)

FIGURE 4: Loop Shaping design procedure.

Step 4. Synthesize a final feedback controller $K_{\text {fin }}(s)$ for the nominal plant $G(s)$ by

$$
K_{\text {fin }}(s)=K_{\text {sh }}(s) V_{1}(s),
$$

where $K_{\mathrm{sh}}(s)$ is given in (7). For tuning purpose, we always choose $V_{2}(s)=I$; then

$$
K_{\text {fin }}(s)=V_{1}(s) K(s) V_{1}(s) .
$$

Step 5. Verify that the desired closed-loop system responses are met. If yes stop. Else adjust $V_{1}(s)$ and $V_{2}(s)$ and go to Step 2.

Remark 9. If $G(s)$ is a nonsquare matrix such as $p>m$ or $p<$ $m$, then it is obvious that the proposed algorithm does not hold. Some minor modifications are required to tackle this problem. In fact, compatible dimensions for the shaped plant $G_{\text {sh }}(s)$ and the shaped controller $K_{\text {sh }}(s)$ require taking $V_{1}(s)$, $V_{2}(s), G(s)$, and $K(s)$ with the same dimension $(\max (p, m)) \times$ $(\max (p, m))$. Thereby, the dimensions of $G_{\mathrm{sh}}(s)$ and $K_{\mathrm{sh}}(s)$ are chosen to be equal to $(\max (p, m)) \times(\max (p, m))$ and the proposed Loop Shaping design procedure still holds.

Remark 10. Note that the final PI controller designed in Algorithm 8 is related to the PI controller designed in Algorithm 6. Indeed, $K_{\text {fin }}(s)$, given by relation (64), is designed, on one hand, using the full MIMO PI controller $K(s)$ given by relation (3) computed via Algorithm 6, and, on the other hand, using weighting functions $V_{1}(s)$ properly designed following Algorithm 8. 
Remark 11. It must be noted that there are severe limitations when the conventional Loop Shaping design procedure is used for MIMO systems as discussed in [20]. Among these limitations, it may still be much harder to find a stabilizing $K_{\text {sh }}$ if $G_{\text {sh }}$ for non-minimum phase or unstable systems. However, this paper succeeds in overcoming these limitations thanks to the first stage of the design procedure that guarantees internally stable closed-loop.

\section{Application}

In this section, simulation results will be performed using three typical examples: the distillation column (unstable system), the ISP reactor (stable system), and the 4-tank process (non-minimum phase system). Furthermore, we will illustrate the superiority of the proposed approach over related ones for set-point tracking, disturbance rejection, and parametric uncertainties scenarios. The comparative study will be established between the following approaches:

(i) The proposed PI controller designed via the Algorithms 6 and 8 .

(ii) The PI controller designed via Algorithm 6.

(iii) The PID controller designed in [14].

(iv) IMC-PI controller approach [13]

(v) The conventional IMC-PID approach [10]

Sedumi and Yalmip Toolbox [30] are used to solve ILMIs. To evaluate the closed-loop performances, the Integral Absolute Error (IAE) and the Total Variation (TV) criteria are considered. They are defined, respectively, by Vu and Lee [13] as follows:

$$
\begin{aligned}
\mathrm{IAE} & =\int_{0}^{T}|e(t)| d t, \\
\mathrm{TV} & =\sum_{k=1}^{T}|u(k+1)-u(k)|,
\end{aligned}
$$

where $T$ is finite time chosen for the integral approach steady state value and $e(t)$ is defined as the total error between the set-points and the outputs.

For the different simulations, unit step changes in the setpoints and disturbances are made to the 1st and 2nd loops. Furthermore, the robustness of the controller is evaluated by considering a perturbation uncertainty of $\pm 10 \%$ in the important parameters, particularity, gains, and delays of the process.

Just for the second example, we will prove that the most conventional multiloop IMC-PID control approach proposed by Economou and Morari [10] fails and that the multiloop IMC-PI proposed by $\mathrm{Vu}$ and Lee [13] has less TV performances. This last approach will not be tested on the third example since it is only appropriate for first-order systems.

For systems with given transfer matrix, the passage from the matrix transfer to a minimal state space model is established using Gilbert method detailed in [31].
For the orthogonal collocation method, optimal parameters are chosen such as $N=3$ and $p=q=-1 / 2$. The different performances singular values are plotted by means of $1 / \bar{\sigma}(S)$ and $1 / \bar{\sigma}\left(S_{\text {sh }}\right)$ by noting that $\max 1 / \bar{\sigma}\left(S_{\text {sh }}\right)$ is equal to $\min \bar{\sigma}\left(S_{\mathrm{sh}}\right)$.

To prove the validity of the transformation between the state space representation and the corresponding transfer matrix and the approximation of the delayed system, let us introduce the following errors: let $e_{1}$ be the error between the unit step response to the state space representation (1) and the corresponding transfer matrix of the LTI MIMO with multiple time delays $G(s) . e_{2}$ is defined as the total error between the delayed system (1) outputs and the approximated ones by model (4)-(5) using the orthogonal collocation method.

Remark 12. Due the bad performances obtained via the PID controller designed via the approach given in [14], an additive filter is joined to the derivative action to attenuate noises. Thus, the transfer matrix of the PID controller with filter considered is described by

$$
\begin{aligned}
& K_{\mathrm{PID}, f}(s)=F_{1, \mathrm{PID}}+\frac{F_{2, \mathrm{PID}}}{s}+F_{3, \mathrm{PID}} \times \frac{s}{\tau_{d} s+1}, \\
& \tau_{d}>0 .
\end{aligned}
$$

It should be noted that the PID controller with filter (66) is applied to stable and non-minimum phase systems. Due to bad simulation results performed for the unstable system, the PID controller with filter is not considered.

5.1. Example 1: The Distillation Column System. Consider the typical example of the distillation column described in $[6,32$, 33] belonging to the class of MIMO unstable plants with input delays; its transfer matrix model is described by Mete et al. [32] as follows:

$$
G(s)=\left[\begin{array}{cc}
\frac{3.0400 e^{-\tau_{2} s}}{s} & \frac{-278.2000 e^{-\tau_{3} s}}{s(s+6)(s+30)} \\
\frac{0.0520 e^{-\tau_{2} s}}{s} & \frac{206.6000 e^{-\tau_{3} s}}{(s+6)(s+30)}
\end{array}\right] .
$$

Applying a column decomposition method [31] for (67), the state space representation (1) can be deduced as follows:

$$
\begin{aligned}
& A_{0}=\left(\begin{array}{cccc}
0 & 0 & 0 & 0 \\
0 & 0 & 0 & 0 \\
0 & 0 & -6 & 0 \\
0 & 0 & 0 & -30
\end{array}\right), \\
& B_{0}=\left(\begin{array}{ll}
1 & 0 \\
0 & 0 \\
0 & 0 \\
0 & 0
\end{array}\right),
\end{aligned}
$$




$$
\begin{aligned}
B_{1} & =\left(\begin{array}{ll}
0 & 0 \\
0 & 1 \\
0 & 1 \\
0 & 1
\end{array}\right), \\
C & =\left(\begin{array}{llll}
3.0400 & -1.5400 & 1.9300 & 0.3800 \\
0.0520 & 1.1400 & -1.4300 & 0.2800
\end{array}\right)
\end{aligned}
$$

for $\tau_{2}=0.5 \mathrm{~h}$ and $\tau_{3}=0.6 \mathrm{~h}$.

Using the orthogonal collocation method, the following matrices are obtained for model (4)-(5):

$$
\begin{aligned}
& \bar{A}_{2}=\bar{A}_{3} \\
& =\left(\begin{array}{cccc}
10.3923 & 1.1547 & -1.1547 & 0.8038 \\
-4.6188 & -0.0000 & 4.6188 & -3 \\
1.1547 & -1.1547 & -10.3923 & 11.1962 \\
-1.4291 & 1.3333 & -19.9043 & 19
\end{array}\right) \text {, } \\
& \bar{B}_{2}=\bar{B}_{3}=\left(\begin{array}{cc}
-11.1962 & -11.1962 \\
3 & 3 \\
-0.8038 & -0.8038 \\
1 & 1
\end{array}\right) \text {, } \\
& \bar{C}_{2}=\bar{C}_{3}=\left(\begin{array}{llll}
0 & 0 & 0 & 1 \\
0 & 0 & 0 & 1
\end{array}\right) .
\end{aligned}
$$

By solving Algorithm 6 with $X_{1}=I_{14}+0.0010$ and $\delta=0.1000$ yielding to $\alpha=1.5000$, the following PI gains are obtained:

$$
\begin{aligned}
& F_{1}=\left(\begin{array}{ll}
0.1945 & 0.2953 \\
0.1946 & 0.2955
\end{array}\right), \\
& F_{2}=\left(\begin{array}{ll}
0.0432 & 0.0006 \\
0.0429 & 0.0006
\end{array}\right) .
\end{aligned}
$$

Thus, the PI controller transfer matrix is given by

$$
K(s)=\left[\begin{array}{ll}
\frac{0.1945 s+0.0432}{s} & \frac{0.2953 s+0.0006}{s} \\
\frac{0.1946 s+0.0429}{s} & \frac{0.2955 s+0.0006}{s}
\end{array}\right]
$$

Figure 5 illustrates the validity of the passage between the transfer matrix model (67) and the state space representation (1) and also the validity of the approximated model (4)-(5) obtained via the orthogonal collocation method.

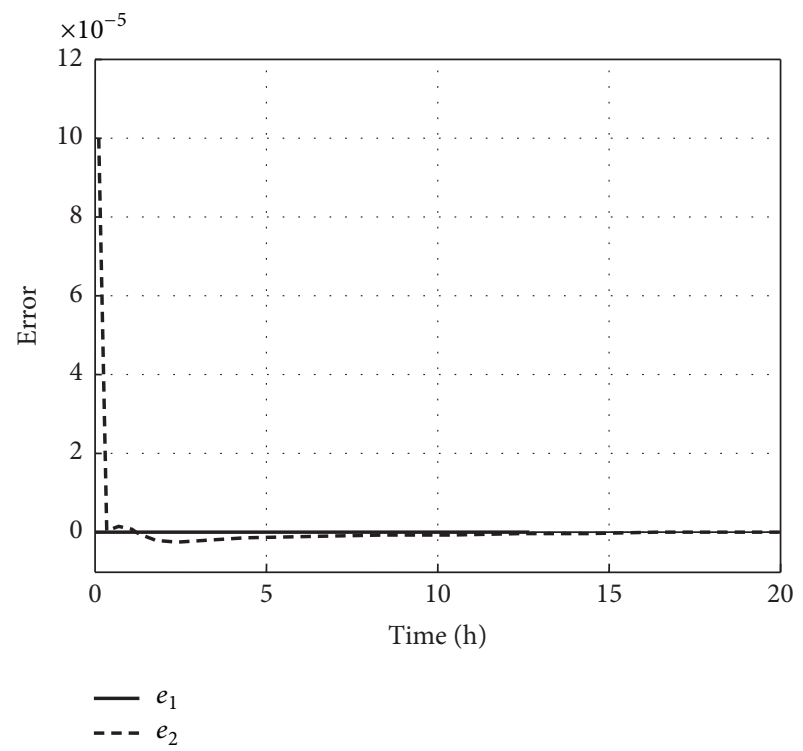

FIGURE 5: Models validation of the distillation column.

TABLE 1: Comparative analysis of controller's performances: the distillation column case study.

\begin{tabular}{lcccc}
\hline \multirow{2}{*}{ Tuning method } & \multicolumn{2}{c}{ Set-point } & \multicolumn{2}{c}{ Disturbance } \\
& IAE & TV & IAE & TV \\
\hline Proposed & 175.16 & 19.82 & 453.14 & 19.77 \\
PI controller & 494.05 & 1.810 & 1366.10 & 6.69 \\
\hline
\end{tabular}

Figure 6 proves that the PI design procedure satisfies the desired specifications for a precompensator $V_{1}(s)$ and postcompensator $V_{2}(s)$ chosen as follows:

$$
\begin{aligned}
& V_{1}(s)=\left[\begin{array}{cc}
1.8000 \frac{s+1}{s+1.0400} & 0 \\
0 & 2 \frac{s+1}{s+1.0200}
\end{array}\right], \\
& V_{2}(s)=\left[\begin{array}{ll}
1 & 0 \\
0 & 1
\end{array}\right] .
\end{aligned}
$$

To boost the low frequency gain and give almost zero steady state error, $V_{1}(s)$ is chosen accordingly as an approximated PI precompensator.

The resulting performance indices for the proposed multivariable controller and the one computed by ILMI method for the nominal and perturbed system cases are summarized in Tables 1 and 2. The proposed controller affords better performances especially for the second output and better disturbance rejection over PI controller as shown by Figures 8 and 9. As listed in Tables 1 and 2, the controller settings of the proposed method provide superior performances by the smallest total IAE for both case studies: set-point changes, disturbances changes, and parametric uncertainties. Acceptable TV indices are also shown by the proposed method for this process. 

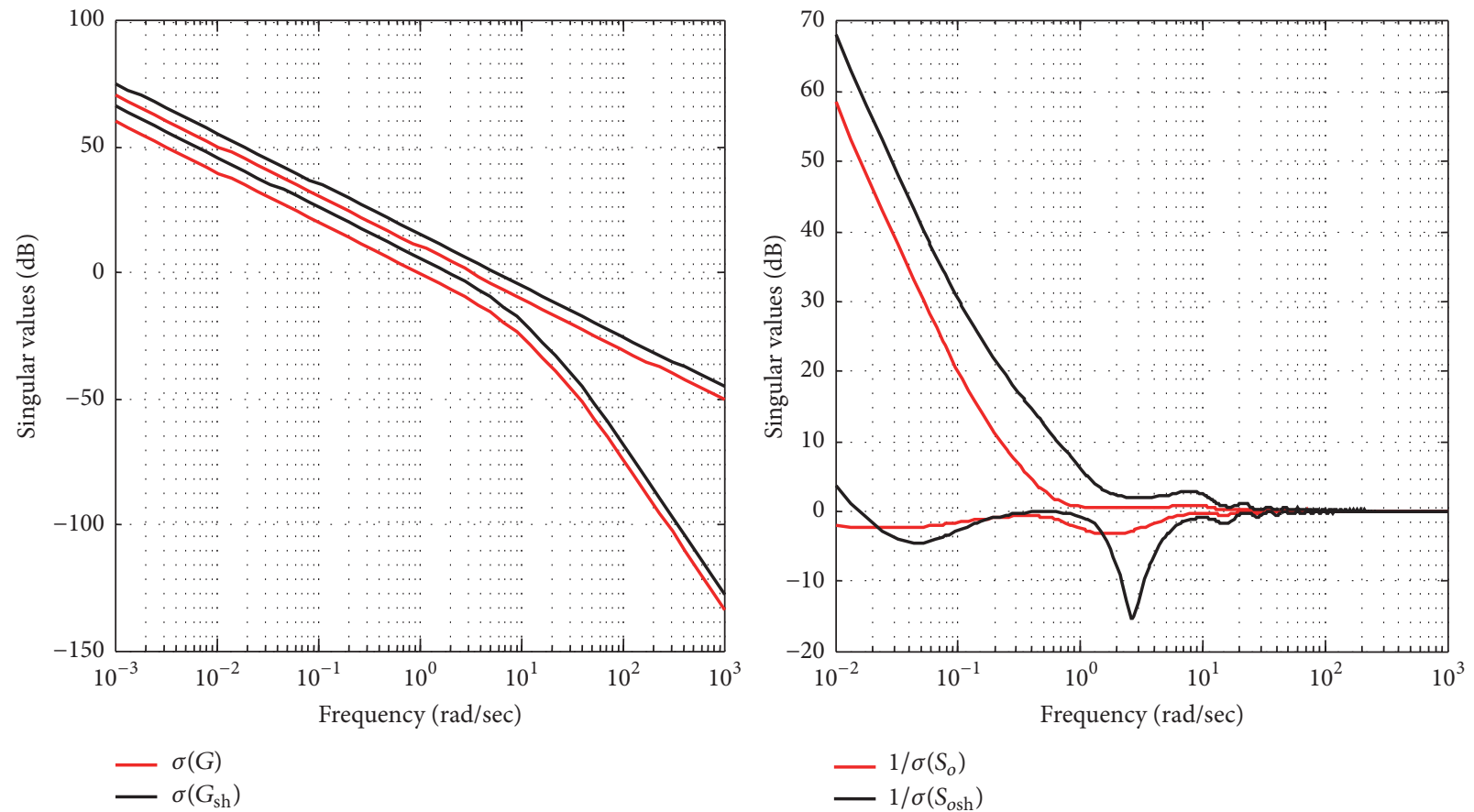

FIgURE 6: Singular values of $G, G_{\text {sh }}, 1 / S_{o}$, and $1 / S_{\text {osh }}$ for the distillation column.

TABLE 2: Robustness analysis under $\pm 10 \%$ in the gain and delay: the distillation column with input delays.

\begin{tabular}{lcccccccc}
\hline & \multicolumn{3}{c}{ The distillation column with input delays $(+10 \%)$} & \multicolumn{3}{c}{ The distillation column with input delays } \\
Tuning method & \multicolumn{2}{c}{ Set-point } & \multicolumn{2}{c}{ Disturbance } & \multicolumn{3}{c}{ Set-point } & Disturbance \\
& IAE & TV & IAE & TV & IAE & TV & IAE & TV \\
& 166.17 & 60.42 & 453.14 & 19.77 & 189.34 & 11.97 & 371.23 & 15.83 \\
Proposed & 457.45 & 1.82 & 1366.10 & 6.69 & 537.84 & 1.80 & 1118 & 5.18 \\
PI controller & & & & & &
\end{tabular}

5.2. Example 2: The Industrial Scale Polymerization (ISP) Reactor. Consider the ISP reactor system described by its transfer matrix given by Chien et al. [34] as follows:

$$
G(s)=\left[\begin{array}{cc}
\frac{22.8900 e^{-\tau_{2} s}}{4.5720 s+1} & \frac{-11.6400 e^{-\tau_{3} s}}{1.8070 s+1} \\
\frac{4.6890 e^{-\tau_{2} s}}{2.1740 s+1} & \frac{5.8000 e^{-\tau_{3} s}}{1.8010 s+1}
\end{array}\right] .
$$

Its minimal realization via Gilbert method gives the state space model (1) where

$$
\begin{aligned}
A_{0} & =\left(\begin{array}{cccc}
-0.2187 & 0 & 0 & 0 \\
0 & -0.5534 & 0 & 0 \\
0 & 0 & -0.4600 & 0 \\
0 & 0 & 0 & -0.5552
\end{array}\right), \\
B_{0} & =\left(\begin{array}{cc}
5.0065 & 0 \\
0 & 0 \\
2.1569 & 0 \\
0 & 0
\end{array}\right),
\end{aligned}
$$

$$
\begin{aligned}
B_{1} & =\left(\begin{array}{cc}
0 & 0 \\
0 & -6.4416 \\
0 & 0 \\
0 & 3.2204
\end{array}\right), \\
C & =\left(\begin{array}{llll}
1 & 1 & 0 & 0 \\
0 & 0 & 1 & 1
\end{array}\right)
\end{aligned}
$$

for $\tau_{2}=0.2 \mathrm{~h}$ and $\tau_{3}=0.4 \mathrm{~h}$.

Let us first test for the previous system the conventional IMC-PID approach proposed by Economou \& Morari [10]. In this approach, the IMC interaction measure surfaces are practical tools to assess the potential value of the multiloop design. By their definitions, they must vary between 0 and 1 such that for the $i$ th input/output pair of a particular system configuration, the Row IMC interaction measure $R_{i}$ is the quantity defined by

$$
R_{i}(i \omega) \triangleq \frac{1}{1+f_{R_{i}}^{*}(i \omega)}=\frac{\sum_{j, j \neq i} g_{i j}(i \omega)}{\sum_{j}\left|g_{i j}(i \omega)\right|}, \quad 0 \leq \omega<\infty
$$


TABLE 3: Interaction measures of the ISP reactor via the IMC-PID approach [10].

\begin{tabular}{lrr}
\hline Pairing & \multicolumn{1}{c}{ IMC interaction measure } \\
\hline$\left(u_{1}, y_{1}\right)=(1,1)$ & $R_{i}(i \omega)=\frac{\left|g_{12}\right|}{\left|g_{11}\right|+\left|g_{12}\right|}$ & $C_{i}(i \omega)=\frac{\left|g_{21}\right|}{\left|g_{11}\right|+\left|g_{21}\right|}$ \\
$\left(u_{2}, y_{2}\right)=(2,2)$ & $R_{i}(i \omega)=\frac{\left|g_{21}\right|}{\left|g_{21}\right|+\left|g_{22}\right|}$ & $C_{i}(i \omega)=\frac{\left|g_{12}\right|}{\left|g_{12}\right|+\left|g_{22}\right|}$ \\
$\left(u_{2}, y_{1}\right)=(1,2)$ & $R_{i}(i \omega)=\frac{\left|g_{11}\right|}{\left|g_{11}\right|+\left|g_{12}\right|}$ & $C_{i}(i \omega)=\frac{\left|g_{22}\right|}{\left|g_{12}\right|+\left|g_{22}\right|}$ \\
$\left(u_{1}, y_{2}\right)=(2,1)$ & $R_{i}(i \omega)=\frac{\left|g_{22}\right|}{\left|g_{21}\right|+\left|g_{22}\right|}$ & $C_{i}(i \omega)=\frac{\left|g_{11}\right|}{\left|g_{11}\right|+\left|g_{21}\right|}$
\end{tabular}

whereas, for the same input/output pair and configuration, the complementary quantity $C_{i}$ is defined by

$$
C_{i}(i \omega) \triangleq \frac{1}{1+f_{C_{i}}^{*}(i \omega)}=\frac{\sum_{j \neq i} g_{j i}(i \omega)}{\sum_{j}\left|g_{j i}(i \omega)\right|}, \quad 0 \leq \omega<\infty
$$

The following scenarios are expected:

(i) $0.5<R_{i}, C_{i}<1.0$ corresponds to significant interactions between the multiple loops and an overall poor performances of the multiloop structure are expected.

(ii) $0<R_{i}, C_{i} \leq 0.5$ corresponds to good pairing and SISO controllers can be designed for each loop and granting good performances.

For the ISP reactor, the transfer function matrix (73) can be written as follows:

$$
\begin{aligned}
G(s) & =\left[\frac{y}{u}\right]=\left[\begin{array}{ll}
\frac{22.8900 e^{-0.2 s}}{4.5720 s+1} & \frac{-11.6400 e^{-0.4 s}}{1.8070 s+1} \\
\frac{4.6890 e^{-0.2 s}}{2.1740 s+1} & \frac{5.8000 e^{-0.4 s}}{1.8010 s+1}
\end{array}\right] \\
& =\left[\begin{array}{ll}
g_{11} e^{-\theta_{11}} & g_{12} e^{-\theta_{12}} \\
g_{21} e^{-\theta_{21}} & g_{22} e^{-\theta_{22}}
\end{array}\right]
\end{aligned}
$$

From (75) and (76), we compute the IMC interactions measure for each pairing as summarized in Table 3. Figure 10 shows the IMC interaction measure for $u_{1}$ controlling $y_{1}$ and $u_{2}$ controlling $y_{2}$ for the original pairing whereas reverse pairing is shown as the IMC interaction measure for $u_{1}$ controlling $y_{2}$ and $u_{2}$ controlling $y_{1}$. As it can be observed by the IMC measure interaction, the original pairing as the reverse pairing cannot guarantee good performances that is why such an approach fails.

Let us now apply the extended IMC-PI controller approach proposed by $\mathrm{Vu}$ and Lee [13] for the class of TITO multidelay processes with first-order plus delay time
TABLE 4: Multiloop PI controller design of the ISP reactor via the IMC-PI approach [13].

\begin{tabular}{lcc}
\hline Loop & $K_{C i}$ & $K_{I i}$ \\
\hline 1 & 0.4211 & 0.1068 \\
2 & 0.1320 & 0.1121 \\
\hline
\end{tabular}

(FOPDT) systems. From the ISP reactor and referring to [13], the following data are deduced:

$$
\begin{aligned}
& K_{11}=22.8900, \\
& K_{12}=-11.6400, \\
& K_{21}=4.6890, \\
& K_{22}=5.8000, \\
& T_{11}=4.5720, \\
& T_{12}=1.8070, \\
& T_{21}=2.1740, \\
& T_{22}=1.8010, \\
& \theta_{11}=0.2000, \\
& \theta_{12}=0.4000, \\
& \theta_{21}=0.2000, \\
& \theta_{22}=0.4000, \\
& \lambda_{1}=0.0900, \\
& \lambda_{2}=0.6900, \\
& K_{e i}=-0.4111, \\
& T_{e i}=-2.1800 .
\end{aligned}
$$

The steady state relative gain array of the ISP reactor is $\Lambda_{i i}(0)=0.7087<1$, which proves that the closed-loop gain is greater than the open-loop gain. The ISP reactor system does not then exhibit open-loop diagonal dominance. The diagonal PI-multi-loop controller parameters $K_{C i}$ and $K_{I i}$ for each loop $i, i=1,2$ are then designed and given by Table 4 . 


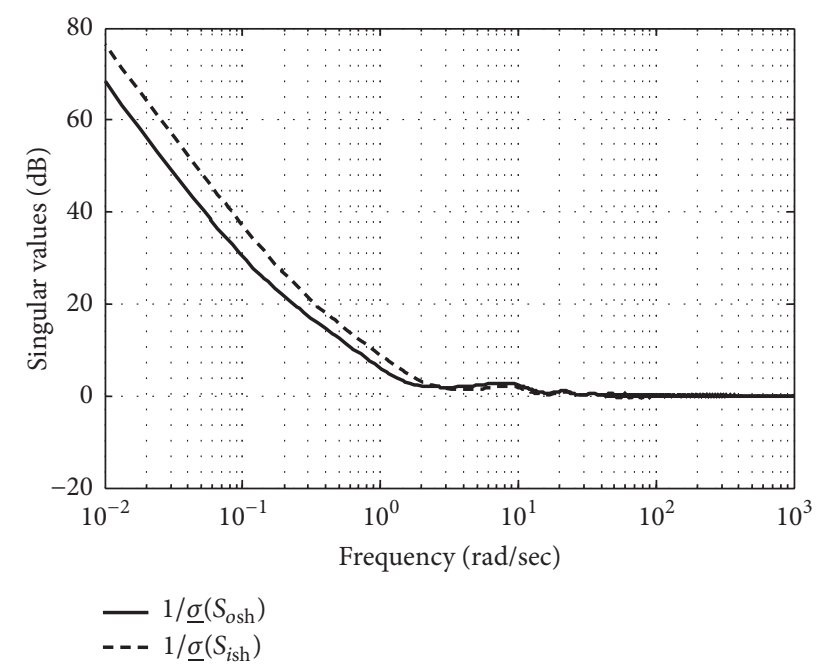

FIGURE 7: Input and output sensitivity matrices of the shaped model for the distillation column.

TABLE 5: Comparative analysis of the controller's performances: the ISP reactor case study.

\begin{tabular}{lcccc}
\hline \multirow{2}{*}{ Tuning method } & \multicolumn{2}{c}{ Set-point } & \multicolumn{2}{c}{ Disturbance } \\
& IAE & TV & IAE & TV \\
\hline Proposed & 20.24 & 10.74 & 123.08 & 2.66 \\
PI & 174.42 & 1.97 & 224.96 & 1.33 \\
PID [14] & 440.57 & 4.93 & 417.66 & 1.48 \\
IMC-PI [13] & 3.97 & 1.98 & 49.05 & 9.13 \\
\hline
\end{tabular}

We have also verified that the two outputs converge to the setpoints in response to a unit step.

For $\tau_{2}=0.2 \mathrm{~h}$ and $\tau_{3}=0.4 \mathrm{~h}$, the reduced model (4)(5) is obtained via the collocation method. Figure 11 proves the validity of models (1) and (4)-(5). By solving Algorithm 6 using the parameters $Y_{1}=I_{18}+0.0020$ and $\delta=0.1000$ and yielding $\alpha=0.7549$, the following PI matrix gains are given:

$$
\begin{aligned}
& F_{1}=\left(\begin{array}{ll}
0.2059 & 0.3061 \\
0.2062 & 0.3060
\end{array}\right), \\
& F_{2}=\left(\begin{array}{ll}
0.0302 & 0.0332 \\
0.0292 & 0.0335
\end{array}\right) .
\end{aligned}
$$

The transfer matrix of the related computed PI controller is given by

$$
K(s)=\left[\begin{array}{ll}
\frac{0.2059 s+0.0302}{s} & \frac{0.3061 s+0.0332}{s} \\
\frac{0.2062 s+0.0292}{s} & \frac{0.3060 s+0.0335}{s}
\end{array}\right] .
$$

By solving algorithm in [14], the PID feedback matrix gains are given by

$$
\begin{aligned}
& F_{1, \mathrm{PID}}=\left(\begin{array}{ll}
0.0187 & 0.2988 \\
0.0192 & 0.2989
\end{array}\right), \\
& F_{2, \mathrm{PID}}=\left(\begin{array}{ll}
-0.0132 & 0.0812 \\
-0.0135 & 0.0812
\end{array}\right), \\
& F_{3, \mathrm{PID}}=\left(\begin{array}{ll}
0.0151 & 0.0661 \\
0.0151 & 0.0660
\end{array}\right) .
\end{aligned}
$$

Figure 12 proves that PI design procedure satisfies the desired specifications for a precompensator $V_{1}(s)$ and postcompensator $V_{2}(s)$ chosen as follows:

$$
\begin{aligned}
& V_{1}(s)=\left[\begin{array}{cc}
3.5000 \times \frac{0.5000 s+1}{s+1} & 0 \\
0 & 3 \times \frac{0.1500 s+1}{s+1}
\end{array}\right], \\
& V_{2}(s)=\left[\begin{array}{ll}
1 & 0 \\
0 & 1
\end{array}\right] .
\end{aligned}
$$

For a sequential unit step change in the set-points at $t=0$ and $t=600 \mathrm{~h}$, one can see that the proposed controller has the faster rising time and settling response over other ILMI approaches as shown by Figure 14. The disturbance model $G_{d}$ is taken as $G_{d}=$ $\left[-4.2430 e^{-0.4 s} /(3.4450 s+1)-0.6010 e^{-0.4 s} /(1.9820 s+1)\right]^{T}$ as in [34]. Unit step changes in the disturbance were also made to the 1st and 2nd loops, respectively, as shown by Figure 15. The resulting performance indices for the nominal and perturbed system cases for various tuning methods are given in Tables 5 and 6. The proposed MIMO PI controller provides superior performances over PI controller and PID controller designed via ILMI approaches by means of the smallest total IAE. Acceptable TV values are also listed by the proposed method.

5.3. Example 3: The 4-Tank Process. Consider the quadrupletank process for which one of the two transmission-zeros of the linearized system dynamics can be moved between the positive and negative real axis [35]. The corresponding model with multiple delays is described in [36] by taking into account transport delays between valves and tanks. Applying the numerical values corresponding to the non-minimum phase model found in [35], system (1) is given by

$$
A_{0}=\left(\begin{array}{cccc}
-0.1993 & 0 & 0 & 0 \\
0 & -0.1422 & 0 & 0 \\
0 & 0 & -0.1230 & 0 \\
0 & 0 & 0 & -0.0873
\end{array}\right)
$$


TABLE 6: Robustness analysis under $\pm 10 \%$ parametric uncertainties: The ISP reactor case study.

\begin{tabular}{lcccccccc}
\hline \multirow{2}{*}{ Tuning method } & \multicolumn{3}{c}{ Set-point } & \multicolumn{2}{c}{ ISP (+10\%) } & \multicolumn{2}{c}{ Disturbance } & \multicolumn{2}{c}{ Set-point } & \multicolumn{2}{c}{ Disturbance } \\
& IAE & TV & IAE & TV & IAE & TV & IAE & TV \\
\hline Proposed & 19.75 & 8.72 & 120.67 & 2.90 & 21.28 & 9.27 & 127.46 & 2.54 \\
PI & 158.68 & 1.95 & 224.06 & 1.33 & 193.50 & 1.94 & 225.85 & 1.33 \\
PID [14] & 404.24 & 4.82 & 415.56 & 1.28 & 482.68 & 4.94 & 418.84 & 1.52 \\
IMC-PI [13] & 4.36 & 2.05 & 48.33 & 8.26 & 3.58 & 1.92 & 49.95 & 10.32 \\
\hline
\end{tabular}

$$
\begin{aligned}
A_{1} & =\left(\begin{array}{cccc}
0 & 0 & 0.1230 & 0 \\
0 & 0 & 0 & 0.0873 \\
0 & 0 & 0 & 0 \\
0 & 0 & 0 & 0
\end{array}\right), \\
B_{0} & =\left(\begin{array}{cc}
0.0482 & 0 \\
0 & 0.0350 \\
0 & 0 \\
0 & 0
\end{array}\right), \\
B_{1} & =\left(\begin{array}{cc}
0 & 0 \\
0 & 0 \\
0 & 0.0775 \\
0.0559 & 0
\end{array}\right) .
\end{aligned}
$$

The measurement level signals $y_{1}$ and $y_{2}$ are $k_{c} h_{1}$ and $k_{c} h_{2}$ where $k_{c}=0.5 \mathrm{~V} / \mathrm{cm}$. The output matrix is then given by

$$
C=\left(\begin{array}{cccc}
0.5000 & 0 & 0 & 0 \\
0 & 0.5000 & 0 & 0
\end{array}\right)
$$

Its transfer matrix is described by

$$
\begin{aligned}
& G(s) \\
& =\left[\begin{array}{cc}
\frac{0.0241 e^{-s \tau_{2}}}{s+0.1993} & \frac{0.0047 e^{-s\left(\tau_{1}+\tau_{3}\right)}}{(s+0.1995)(s+0.1230)} \\
\frac{0.0024 e^{-s\left(\tau_{1}+\tau_{3}\right)}}{(s+0.1422)(s+0.0873)} & \frac{0.0175 e^{-s \tau_{2}}}{s+0.1422}
\end{array}\right] .
\end{aligned}
$$

For simulation results, the constant delays are chosen as follows: $\tau_{1}=5 \mathrm{~s}, \tau_{2}=2 \mathrm{~s}$, and $\tau_{3}=4 \mathrm{~s}$.

To investigate the validity of the 4 -tank process, the different model errors are depicted in Figure 16. By solving Algorithm 6 using the parameters $Y_{1}=I_{18}+0.2050, \delta=$
0.1000, and yielding $\alpha=0.2500$, the following PI gains are given:

$$
\begin{aligned}
& F_{1}=\left(\begin{array}{ll}
0.1569 & 0.1532 \\
0.0236 & 0.0154
\end{array}\right), \\
& F_{2}=\left(\begin{array}{ll}
0.1569 & 0.1532 \\
0.0236 & 0.0154
\end{array}\right) .
\end{aligned}
$$

Thus, the PI controller transfer matrix is given by

$$
K(s)=\left[\begin{array}{cc}
\frac{0.1569 s+0.1569}{s} & \frac{0.1532 s+0.1532}{s} \\
\frac{0.0236 s+0.0236}{s} & \frac{0.0154 s+0.0154}{s}
\end{array}\right] .
$$

The feedback matrix gains designed by solving algorithm in [14] are given by

$$
\begin{aligned}
& F_{1, \mathrm{PID}}=\left(\begin{array}{ll}
0.1562 & 0.1515 \\
0.0231 & 0.0150
\end{array}\right), \\
& F_{2, \mathrm{PID}}=\left(\begin{array}{ll}
0.1562 & 0.1515 \\
0.0231 & 0.0150
\end{array}\right), \\
& F_{3, \mathrm{PID}}=\left(\begin{array}{ll}
-3.7924 & 5.5584 \\
-3.7924 & 5.5584
\end{array}\right) .
\end{aligned}
$$

Figure 17 shows that PI design procedure satisfies the desired specifications for a precompensator $V_{1}(s)$ and postcompensator $V_{2}(s)$ chosen as follows:

$$
\begin{aligned}
& V_{1}(s)=\left[\begin{array}{cc}
3 \times \frac{2 s+1}{s+1} & 0 \\
0 & 3 \times \frac{3 s+1}{s+1}
\end{array}\right], \\
& V_{2}(s)=\left[\begin{array}{ll}
1 & 0 \\
0 & 1
\end{array}\right] .
\end{aligned}
$$

The resulting performance indices for the proposed multivariable controller and those of other ILMI methods for the nominal and perturbed system cases are summarized in Tables 7 and 8 . For a sequential unit step changes in the setpoint and disturbance, Figures 19 and 20 compare the closedloop time responses and controller output responses afforded 

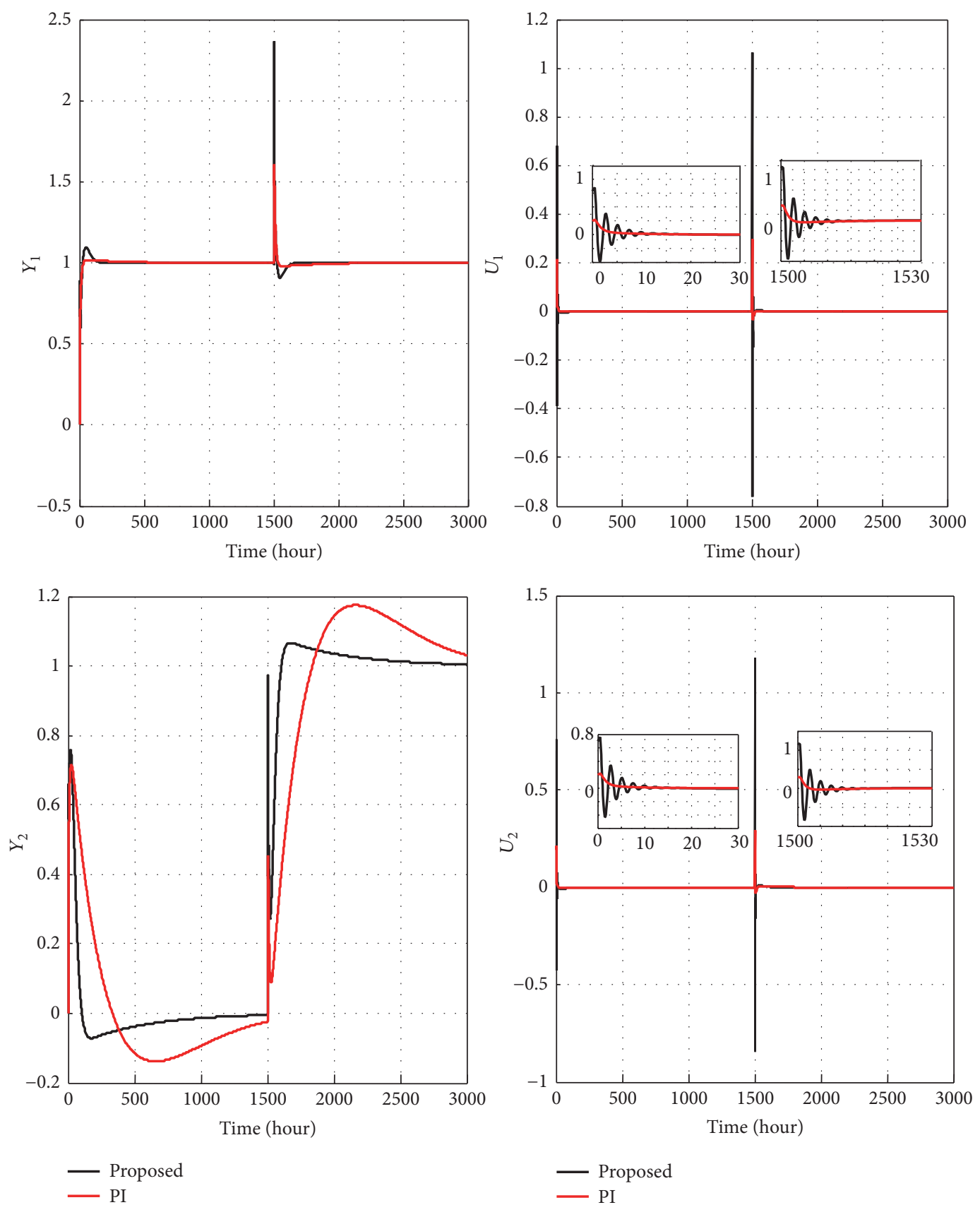

FIGURE 8: Closed-loop responses and controller output responses to set-point changes for the distillation column.

TABLE 7: Comparative analysis of controller's performances. The non-minimum phase 4-tank process.

\begin{tabular}{lccrr}
\hline \multirow{2}{*}{ Tuning method } & \multicolumn{2}{c}{ Set-point } & \multicolumn{2}{c}{ Disturbance } \\
& IAE & TV & IAE & 98.24 \\
Proposed & 479.04 & 119.47 & 105.91 & 15.20 \\
PI & 4005.50 & 39.10 & 105.22 & 11.48 \\
PID [14]. & 4100.80 & 338.06 & 6.75 \\
\hline
\end{tabular}


TABLE 8: Robustness analysis under $\pm 10 \%$ in the gain and delay: the non-minimum phase 4 -tank process case study.

\begin{tabular}{lcccccrrr}
\hline \multirow{2}{*}{ Tuning method } & \multicolumn{3}{c}{ 4-tank process $(+10 \%)$} & \multicolumn{3}{c}{ 4-tank process $(-10 \%)$} \\
& IAE & TV & IAE & TV & IAE & TV & IAE & TV \\
\hline Proposed & 418.26 & 120.51 & 99.43 & 15.84 & 510.06 & 98.66 & 94.85 & 13.21 \\
PI & 3678.10 & 37.14 & 106.96 & 11.99 & 4383.80 & 41.88 & 102.31 & 11.58 \\
PID [14]. & 3768.90 & 333.93 & 106.24 & 7.04 & 4482.50 & 339.06 & 101.65 & 5.76 \\
\hline
\end{tabular}
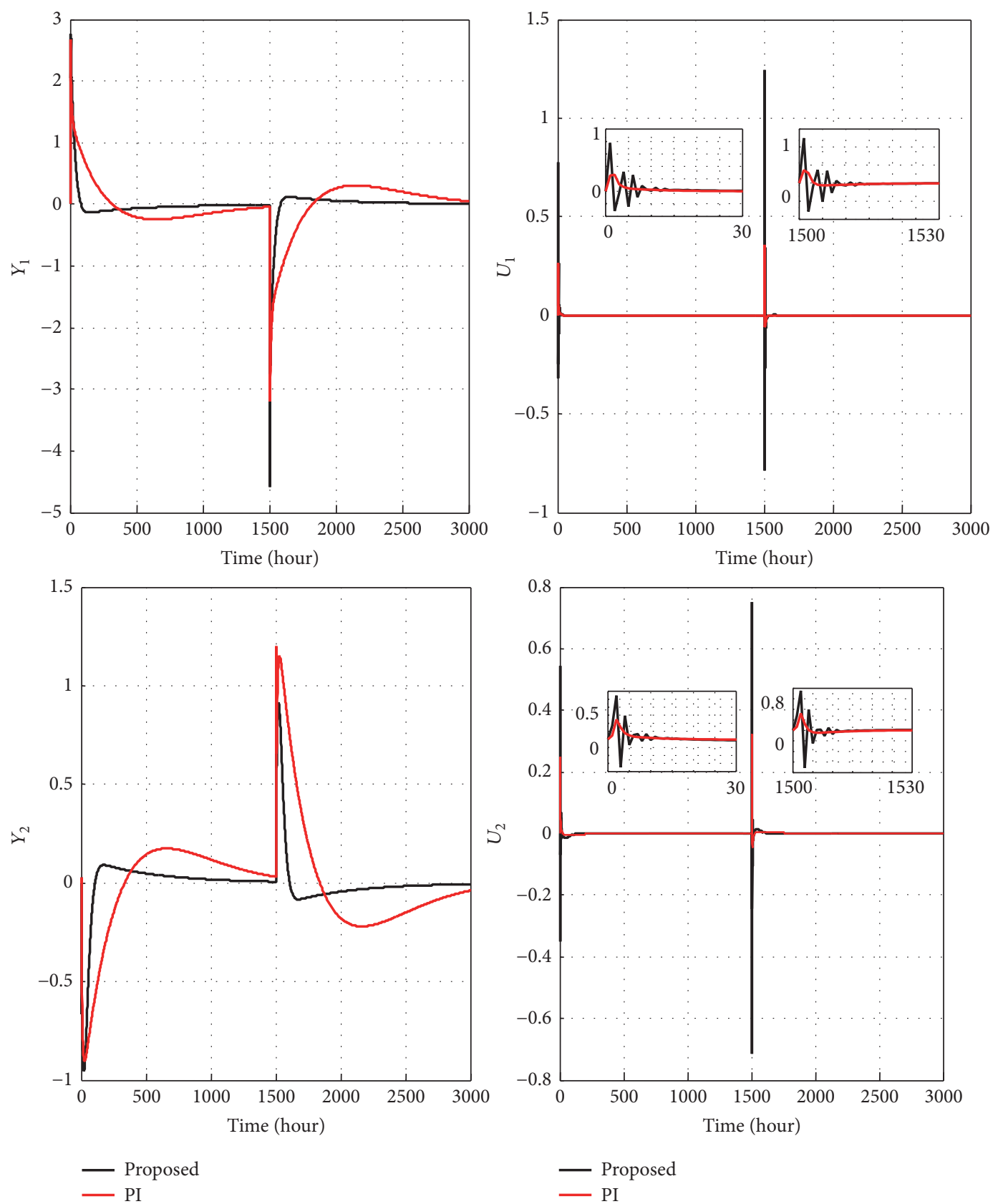

FIGURE 9: Closed-loop responses and controller output responses to unit step changes in the disturbance for the distillation column. 

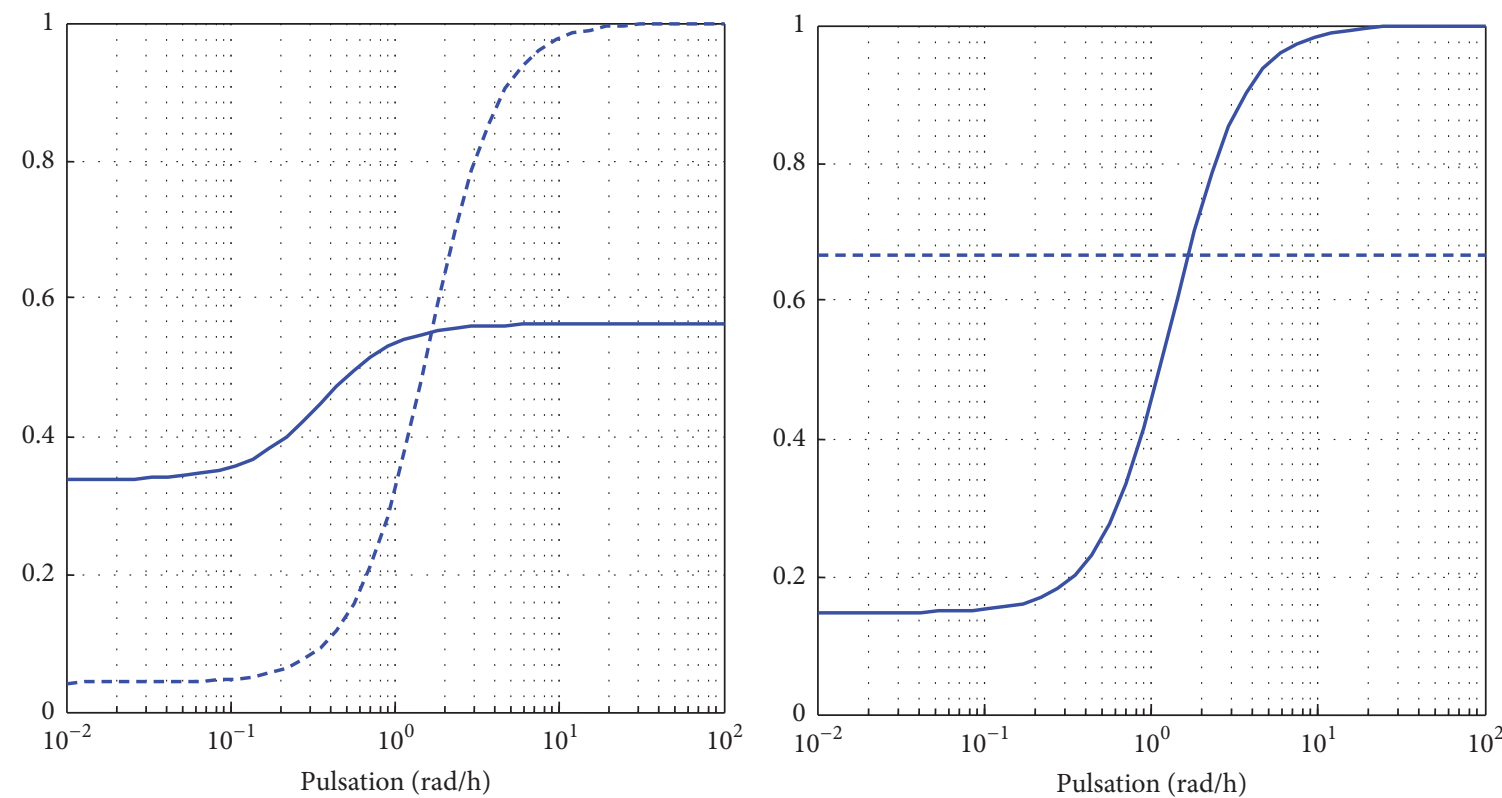

$-R_{i}(i \omega)(1,1)$

--- $C_{i}(i \omega)(1,1)$

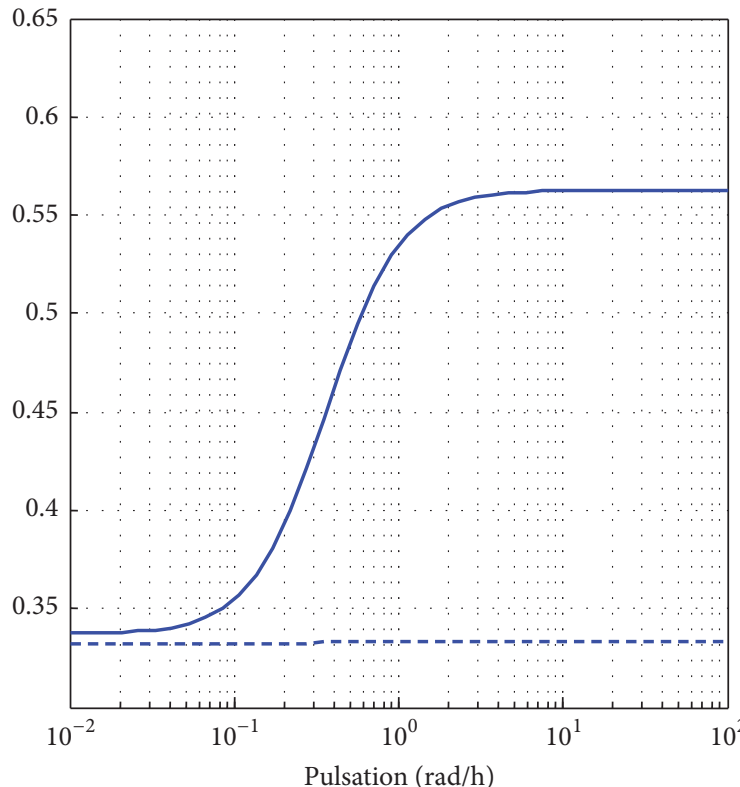

$R_{i}(i \omega)(2,2)$

- - $C_{i}(i \omega)(2,2)$

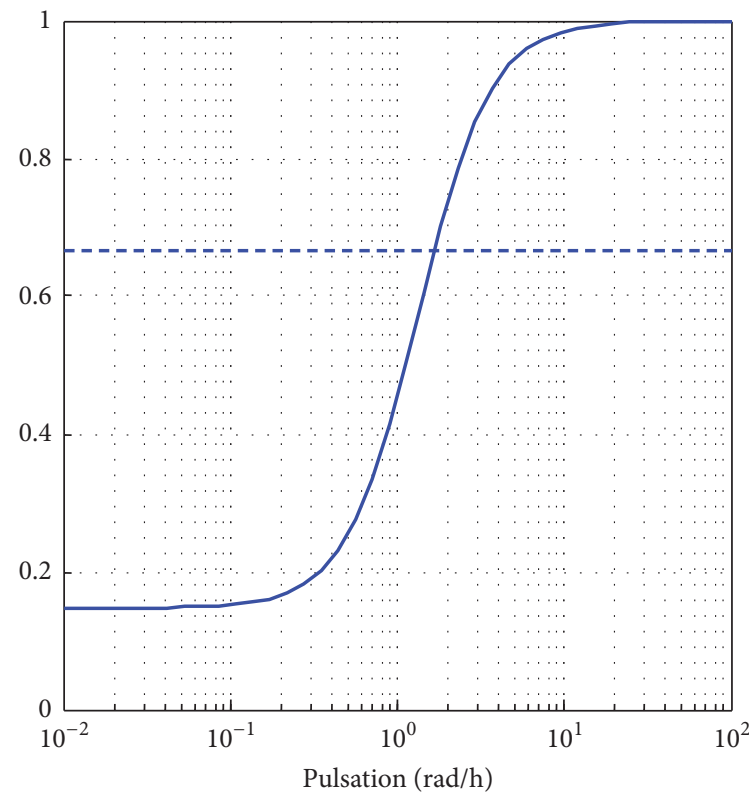

$R_{i}(i \omega)(1,2)$

$R_{i}(i \omega)(2,1)$

- - $C_{i}(i \omega)(1,2)$

- - $C_{i}(i \omega)(2,1)$

FIGURE 10: IMC interaction measures for the ISP reactor: fail of the conventional IMC-PID approach.

by the proposed controller with those given by PI and PID with filter controllers. The disturbance model $G_{d}$ is taken as a perturbation uncertainty of $+10 \%$ in the process gain and time delay into the actual process, simultaneously. As listed in Tables 7 and 8, the proposed MIMO PI controller settings provide superior performance over PI and PID controllers by means of the smallest total IAE. High TV values are explained by non-minimum phase system characteristics for this process.

Furthermore, noise rejection in high frequencies is also known as an important requirement in a control system design. In order to evaluate the effect of such noise on the closed-loop performances of the most complex examples considered in this paper, simulation results have been 


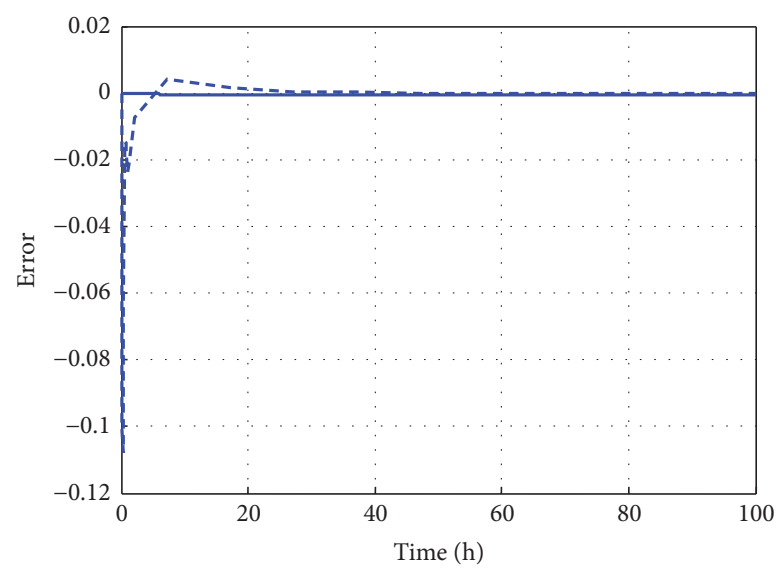

$-e_{1}$

FIGURE 11: Models validation of the ISP reactor.
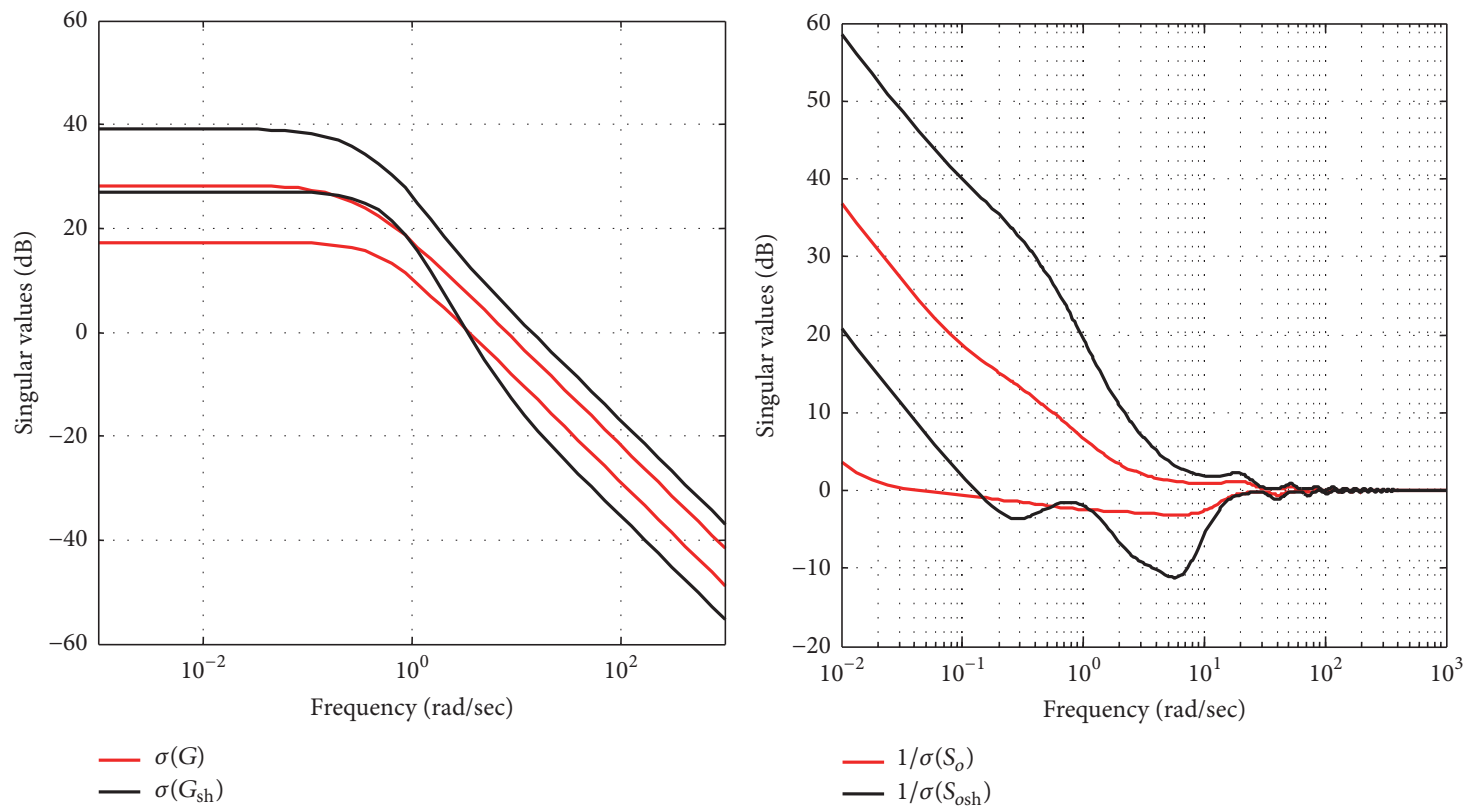

FIGURE 12: Singular values of $G, G_{\mathrm{sh}}, 1 / S_{o}$, and $1 / S_{\text {osh }}$ for the ISP reactor.

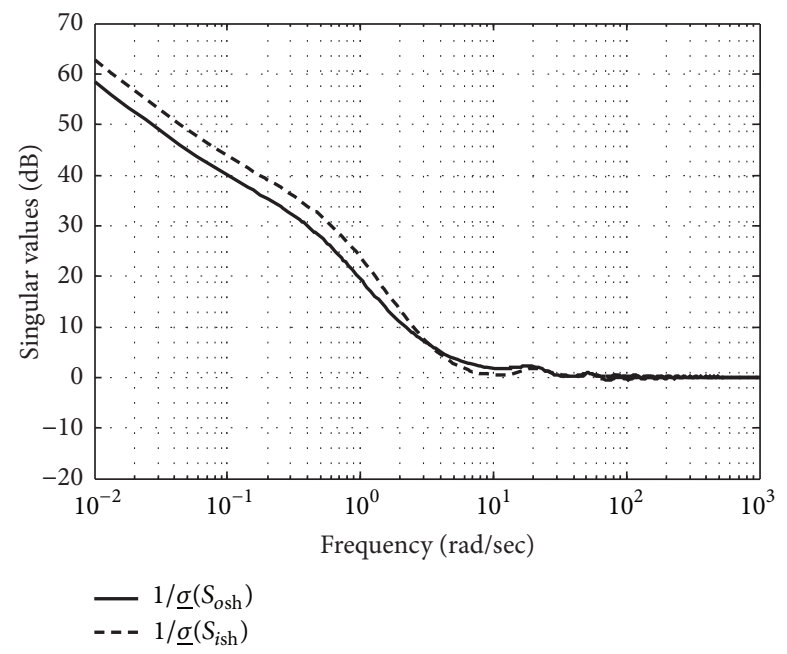

FIGURE 13: Input and output sensitivity matrices of the shaped model for the ISP reactor. 

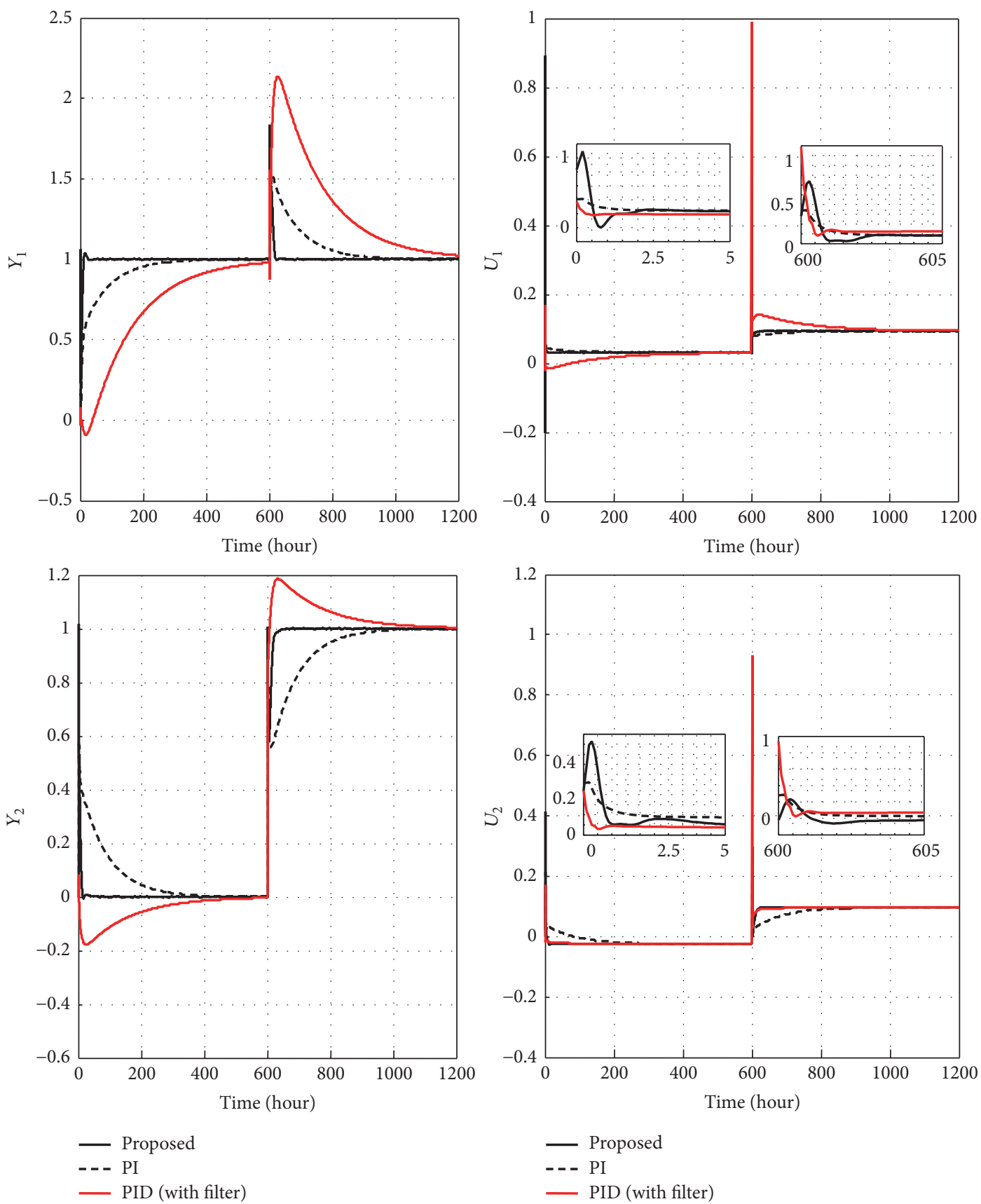

FIGURE 14: Closed-loop responses and controller output responses to set-point changes for the ISP reactor.

conducted taking into account of White Gaussian Noise Measurements (WGNM) with a variation of $0.01 \mathrm{~V}$ and zero mean. It is apparent from Figure 21 that the output responses are not sensitive to WGNM whereas acceptable fluctuations are however observed for the control inputs. Improving such performances will be considered in future works.

Remark 13. Equations (56) and (59) show the effects of the disturbances $d$ and $d_{i}$ on the output vector $y$ and the control signal vector $u_{G}$, respectively. This can be obviously made small by making the output sensitivity function $S_{\text {osh }}$ and the input sensitivity function $S_{i \text { sh }}$ small. In other words, a good disturbance attenuation can be improved by making the sensitivity functions $1 / S_{\text {osh }}$ and $1 / S_{\text {ish }}$ decrease faster in low frequencies. From Figures 7,13 , and 18, it is clear that this principle is well managed for the three examples. Indeed, a high frequency roll-off is shown over $0.01 \mathrm{rad} / \mathrm{s}$ and $1 \mathrm{rad} / \mathrm{s}$ with $31 \mathrm{~dB} /$ decade, $20 \mathrm{~dB} /$ decade and $17.5 \mathrm{~dB} /$ decade for $1 / S_{\text {osh }}$ 

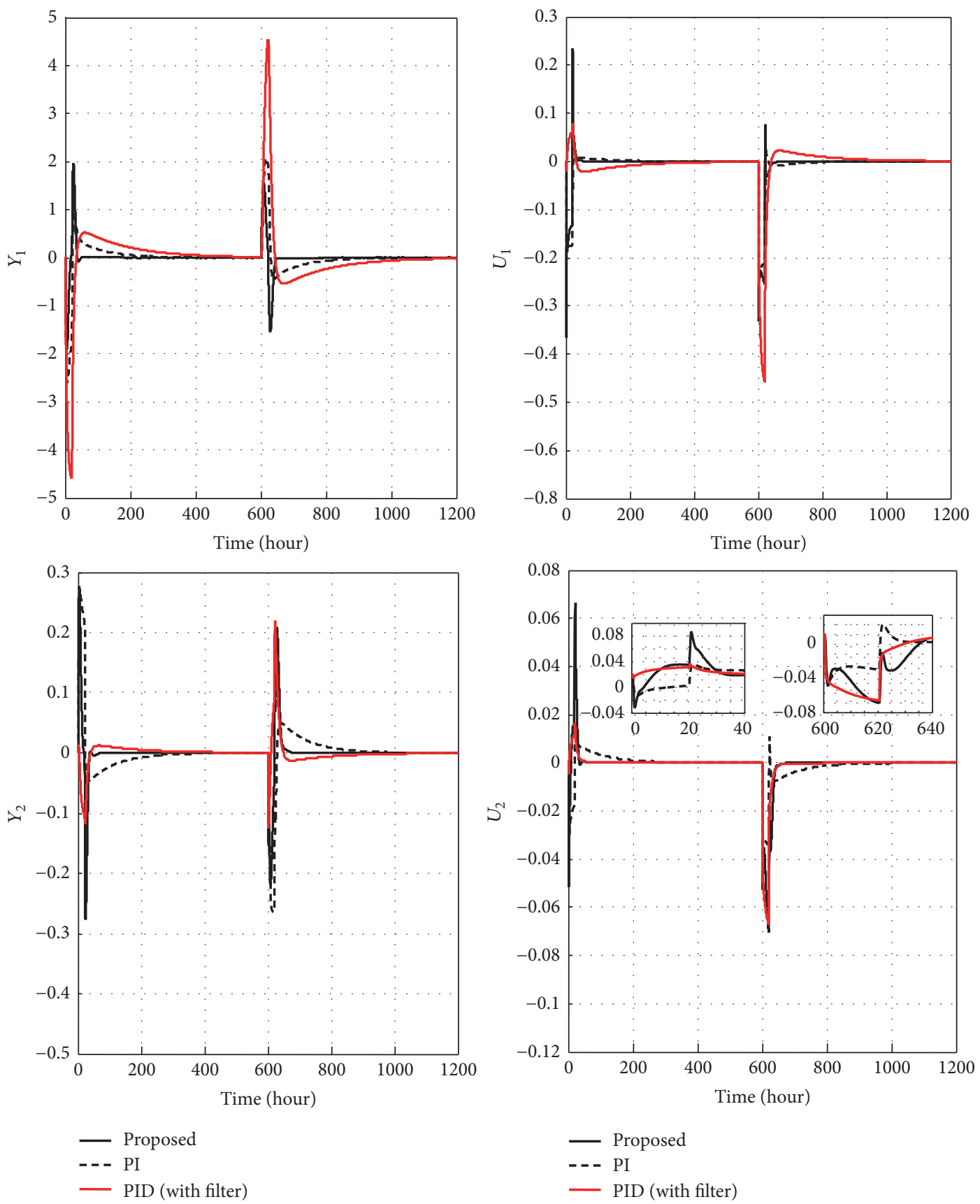

FIGURE 15: Closed-loop responses and controller output responses to unit step changes in the disturbance for the ISP reactor.

and with $33.5 \mathrm{~dB} /$ decade, $20 \mathrm{~dB} /$ decade, and $18 \mathrm{~dB} /$ decade for $1 / S_{i s h}$ for the considered unstable, stable, and non-minimum phase systems, respectively.

5.4. Comparative Analysis. Previous results can be summarized as follows:

(i) Even more the controller design procedure has not considered decoupling principle of multivariable systems; the proposed approach provides generally superior performances by the smallest total IAE for the set-point changes, disturbance changes and parametric uncertainties, over related approaches, for the unstable distillation column, the ISP reactor, and the 4-tank process as summarized by Tables 1-2 and 5-8. Low TV values are also shown for the ISP reactor.

(ii) The proposed method succeeds to synthesize a MIMO PI controller for the ISP reactor when the IMC-PID approach proposed by Economou and Morari [10] fails due to high interactions measure for the original and reverse pairings. Furthermore, the 


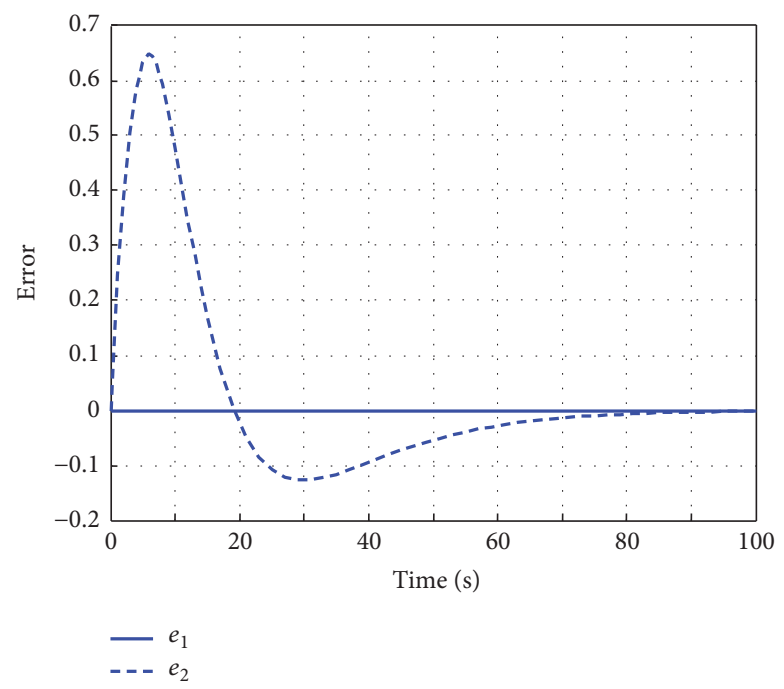

FIGURE 16: Models validation of the 4-tank process.
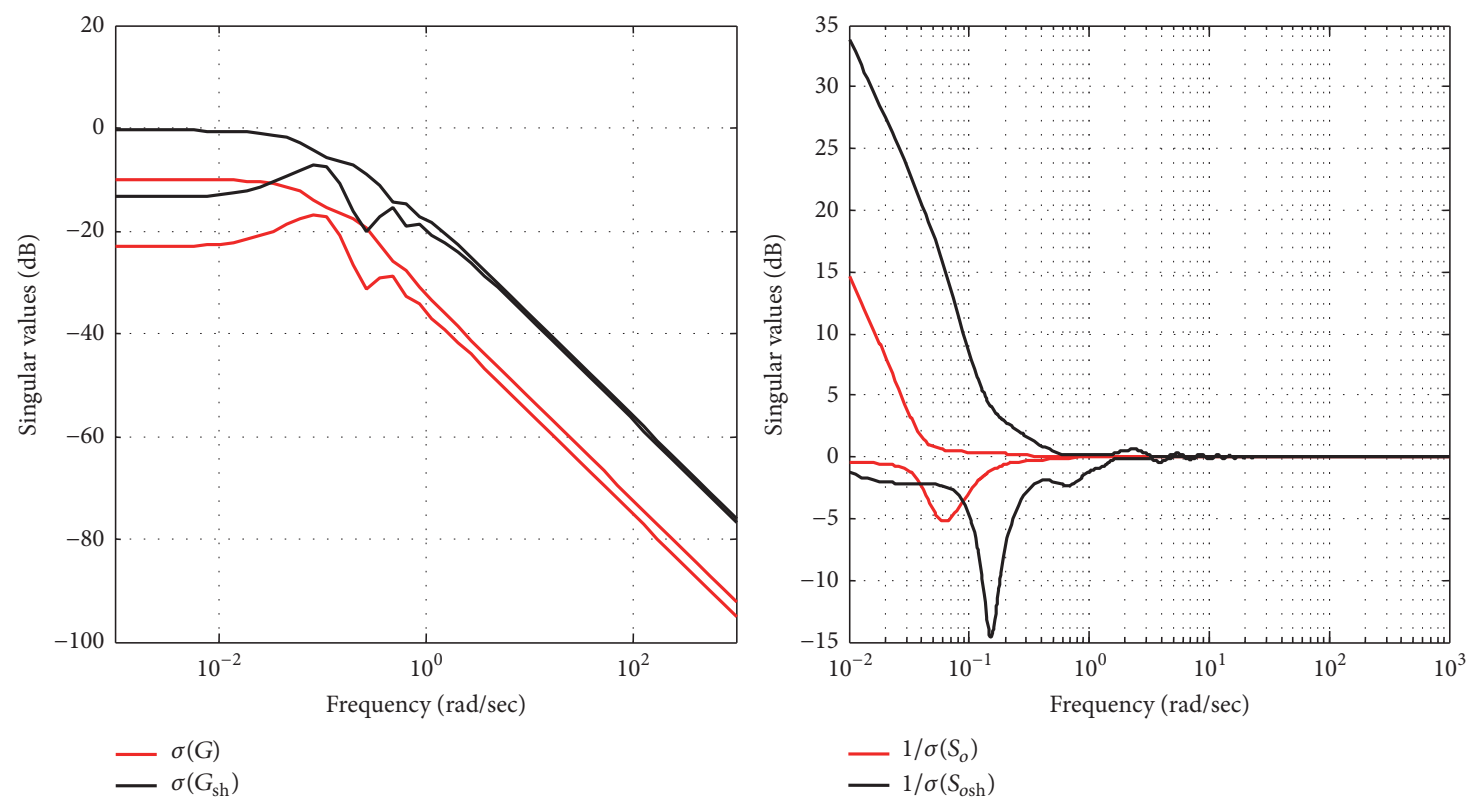

FIGURE 17: Singular values of $G, G_{\text {sh }}, 1 / S_{o}$, and $1 / S_{\text {osh }}$ for the 4 -tank process.

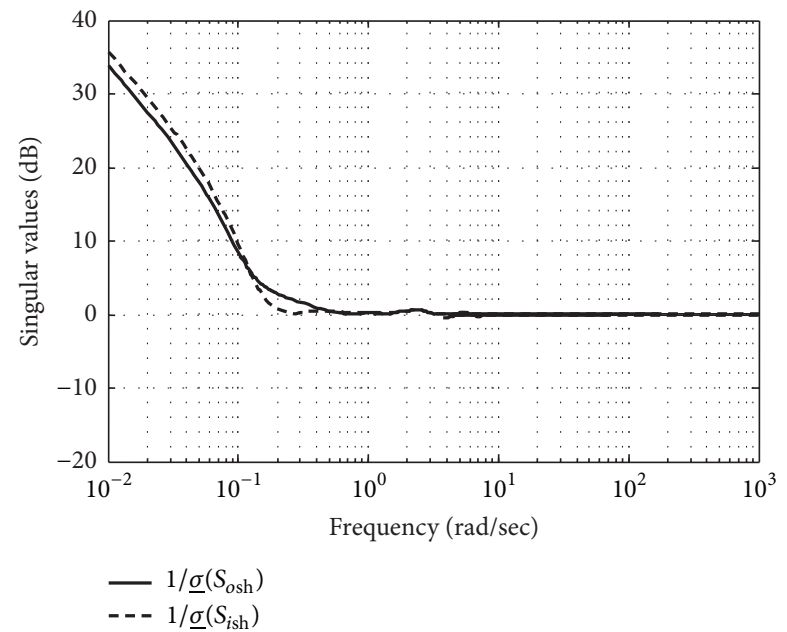

FIGURE 18: Input and output sensitivity matrices of the shaped model for the 4-tank process. 

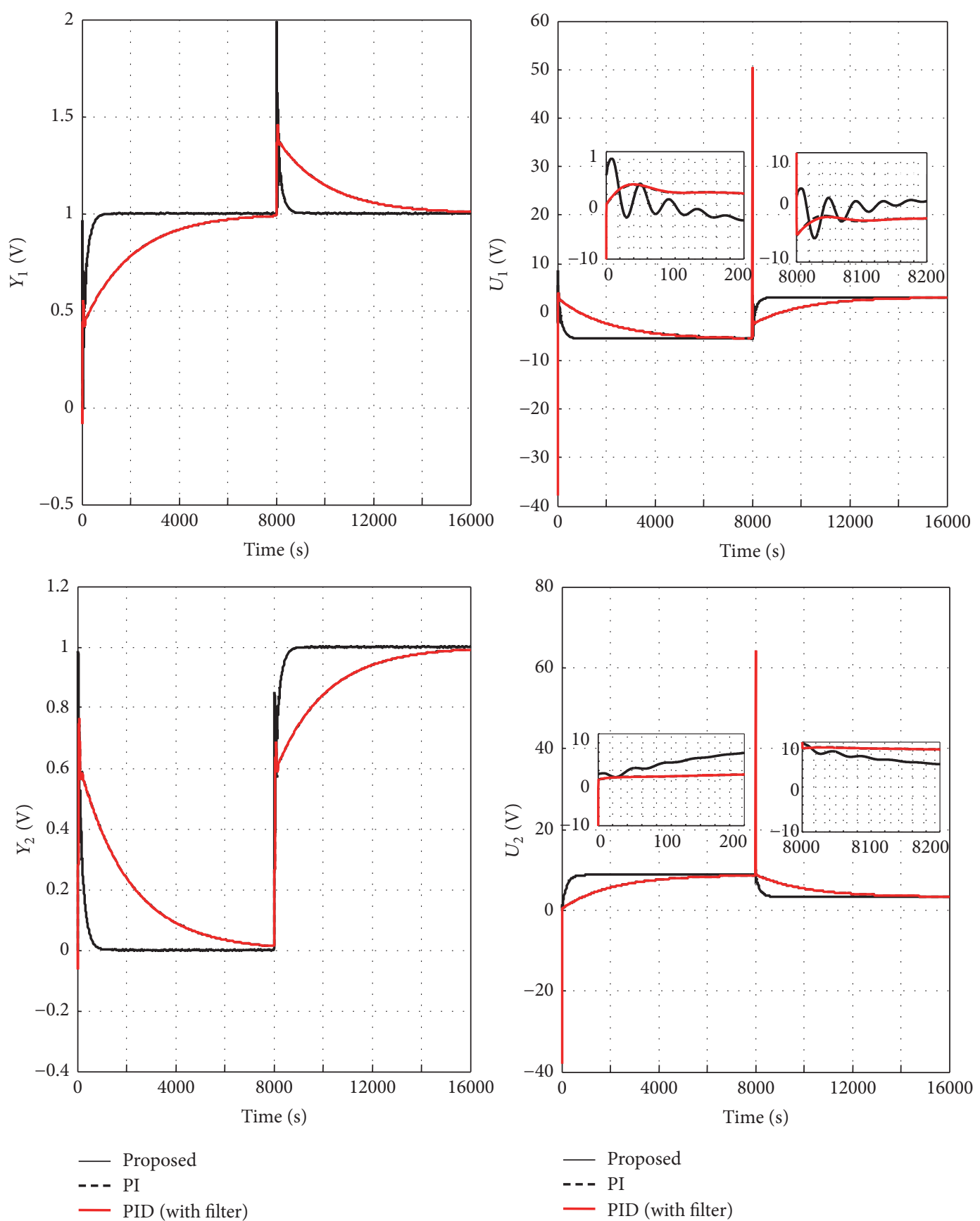

FIGURE 19: Closed-loop responses and controller output responses to set-point changes for the 4-tank process.

proposed controller settings give a much smoother response for the disturbance rejection case with (TV $=2.66, \mathrm{TV}=2.90$ and $\mathrm{TV}=2.54)$ over the IMC-PI approach [13] with $(\mathrm{TV}=9.13, \mathrm{TV}=8.26, \mathrm{TV}=10.32)$ for both the nominal and the perturbed models.

(iii) The proposed method is applicable to the 4-tank process where the IMC-PI approach [13] is not applicable due to the presence of second-order functions in the transfer matrix. The IMC-PI approach proposed by Vu and Lee [13] is only applicable for FOPTD systems.

(iv) The proposed method is applicable to the unstable distillation column with input delays where the IMC-PI approach proposed by Vu and Lee [13] is only applicable for FOPTD systems and where the IMCPID approach proposed by Economou and Morari [10] is limited to models with first or second orders. 

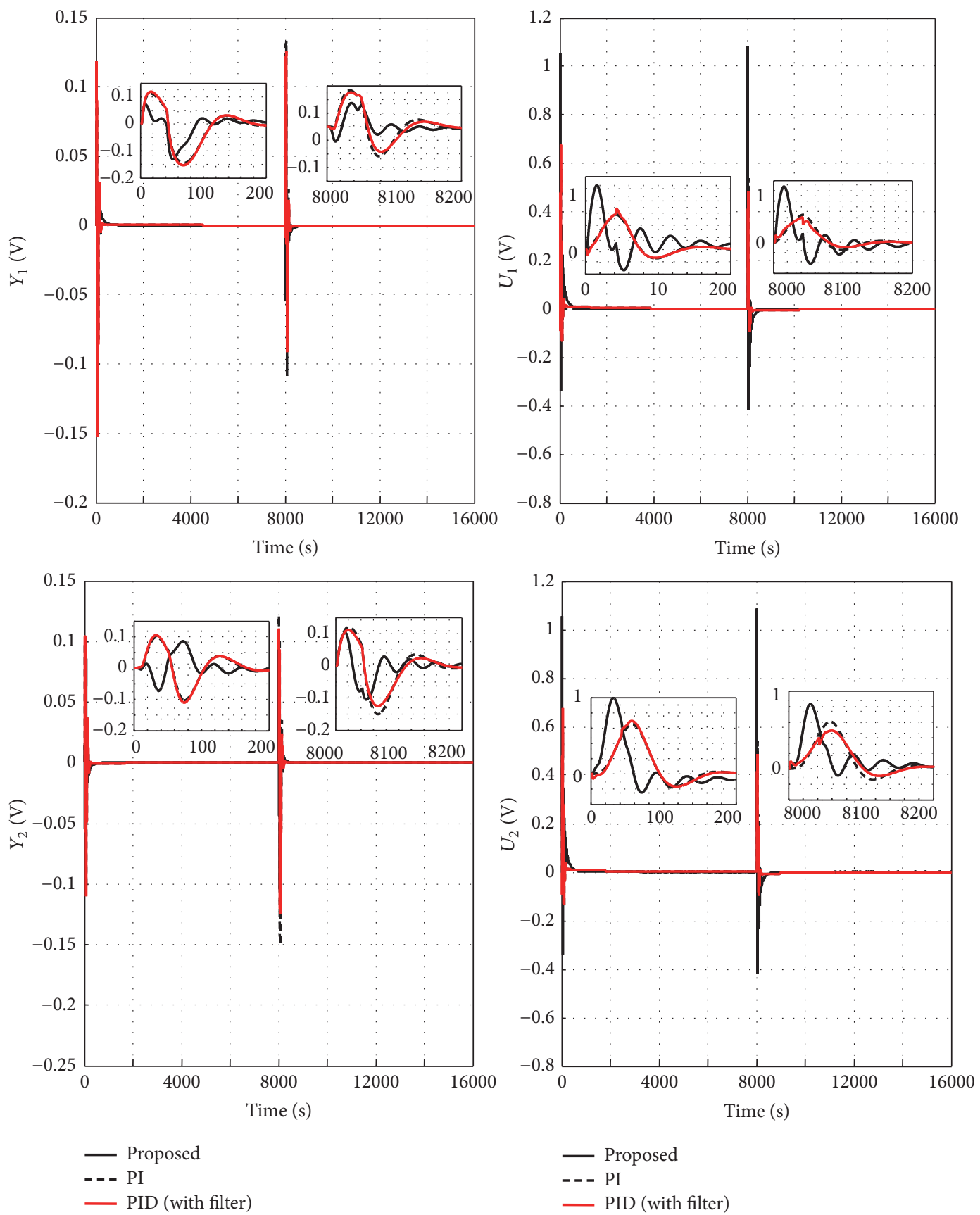

FIGURE 20: Closed-loop responses and controller output responses to unit step changes in the disturbance for the 4-tank process.

It should be pointed out that the unstable distillation column holds third-order elements.

(v) For the 4-tank process, some large IAE and TV values listed are due to non-minimum phase system characteristics; it is obvious that non-minimum zero dynamics cause performance deterioration of the closed-loop system responses (initial undershoot, overshoot and zero crossings) and then increase IAE and TV performances indices.

\section{Conclusion}

This paper presents a MIMO PI controller design procedure for LTI MIMO systems with multiple time delays by means of ILMI and sensitivity functions. The proposed Loop Shaping design procedure with minimizing sensibility functions yields to optimized closed-loop system responses. The distillation column, the ISP reactor, and the 4-tank process as benchmarks of unstable, stable, and non-minimum phase systems are provided to illustrate the validity, effectiveness, 

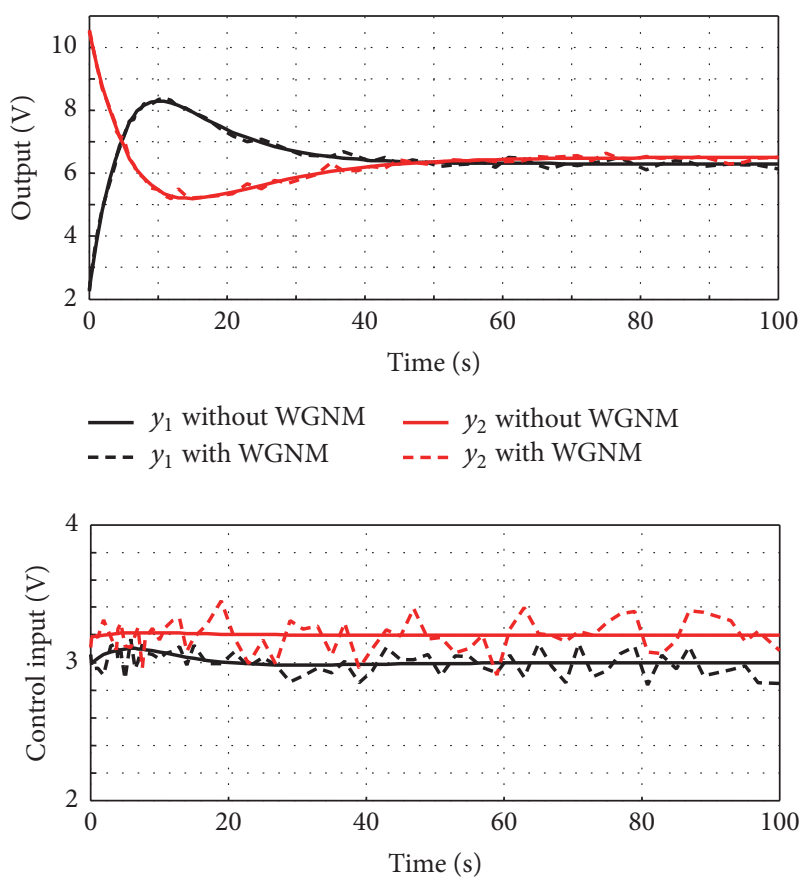

$$
\begin{aligned}
& \text { - } u_{1} \text { without WGNM }-u_{2} \text { without WGNM } \\
& \text {-.- } u_{1} \text { with WGNM _-- } u_{2} \text { with WGNM }
\end{aligned}
$$

FIGURE 21: Closed-loop responses and controller output responses without WGNM and under WGNM.

and robustness of the proposed method. Considering different case studies (set-point tracking, disturbance rejection, and parametric uncertainties), a comparative analysis between the proposed method and related ones showed that the proposed method afforded the superior performances both in the nominal and in the perturbed case studies.

\section{Competing Interests}

The authors declare that there is no conflict of interests regarding the publication of this paper.

\section{References}

[1] K. J. Astrom and T. Hagglund, "Advanced PID control," in ISAThe Instrumentation, Systems, and Automation Society, 2005.

[2] R. Vilanova and A. Visioli, PID Control in the Third Millennium: Lessons Learned and New Approaches, Springer, New York, NY, USA, 2012.

[3] F. D. Bianchi, R. J. Mantz, and C. F. Christiansen, "Multivariable PID control with set-point weighting via BMI optimisation," Automatica, vol. 44, no. 2, pp. 472-478, 2008.

[4] X. Luan, Q. Chen, and F. Liu, "Equivalent transfer function based multi-loop PI control for high dimensional multivariable systems," International Journal of Control, Automation and Systems, vol. 13, no. 2, pp. 346-352, 2015.

[5] S. Boyd, M. Hast, and K. J. Åström, "MIMO PID tuning via iterated LMI restriction," International Journal of Robust and Nonlinear Control, vol. 26, no. 8, pp. 1718-1731, 2016.
[6] A. N. Gündeş, H. Özbay, and A. B. Özgüler, "PID controller synthesis for a class of unstable MIMO plants with I/O delays," Automatica, vol. 43, no. 1, pp. 135-142, 2007.

[7] T. N. L. Vu and M. Lee, "Analytical design of robust multi-loop PI controller for multi-time delay processes," in Advances in Machine Learning and Data Analysis, vol. 48 of Lecture Notes in Electrical Engineering, pp. 95-108, Springer, Berlin, Germany, 2010.

[8] A. N. Gündeş and H. Özbay, "Reliable decentralised control of delayed MIMO plants," International Journal of Control, vol. 83, no. 3, pp. 516-526, 2010.

[9] Q. Jin, F. Hao, and Q. Wang, "IMC-PID controller based on diagonal equivalent model for multivariable systems with multiple time delays," Journal of Chemical Engineering of Japan, vol. 46, no. 3, pp. 209-218, 2013.

[10] C. G. Economou and M. Morari, "Internal Model Control: multi-loop design," Industrial and Engineering Chemistry Process Design and Development, vol. 25, no. 2, pp. 411-419, 1986.

[11] S. Cha, D. Chun, and J. Lee, "Two-step IMC-PID method for multiloop control system design," Industrial and Engineering Chemistry Research, vol. 41, no. 12, pp. 3037-3041, 2002.

[12] M. Lee, K. Lee, C. Kim, and J. Lee, "Analytical design of multiloop PID controllers for desired closed-loop responses," AIChE Journal, vol. 50, no. 7, pp. 1631-1635, 2004.

[13] T. N. L. Vu and M. Lee, "Multi-loop PI controller design based on the direct synthesis for interacting multi-time delay processes," ISA Transactions, vol. 49, no. 1, pp. 79-86, 2010.

[14] F. Zheng, Q.-G. Wang, and T. H. Lee, "On the design of multivariable PID controllers via LMI approach," Automatica, vol. 38, no. 3, pp. 517-526, 2002.

[15] C. Lin, Q.-G. Wang, and T. H. Lee, "An improvement on multivariable PID controller design via iterative LMI approach," Automatica, vol. 40, no. 3, pp. 519-525, 2004.

[16] Y. He and Q. G. Wang, "An improved ILMI method for static output feedback control with application to multivariable PID control," IEEE Transactions on Automatic Control, vol. 51, no. 10, pp. 1678-1683, 2006.

[17] W. Belhaj and O. Boubaker, "On MIMO PID control of the quadruple-tank process via ILMIs approaches: minimum and non-minimum case studies," in Proceedings of the 10th IFAC Symposium on Dynamics and Control of Process Systems (DYCOPS '13), pp. 481-486, Mumbai, India, December 2013.

[18] W. Belhaj and O. Boubaker, "Multivariable PID controller via LMIs: performances assessement," International Journal of Smart Sensing and Intelligent Systems, vol. 8, no. 4, pp. 18961916, 2015.

[19] D. McFarlane and K. Glover, Robust Controller Design Using Normalized Coprime Factor Plant Descriptions, vol. 138 of Lecture Notes in Control and Information Sciences, Springer, Berlin, Germany, 1990.

[20] K. Zhou, J. C. Doyle, and K. Glover, Robust and Optimal Control, Prentice Hall, Upper Saddle River, NJ, USA, 1996.

[21] H. Panagopoulos and K. J. Astrom, "PID control design and loop shaping," in Proceedings of the IEEE International Conference on Control Applications, pp. 103-108, Honolulu, Hawaii, USA, 1999.

[22] W. Tan, T. Chen, and H. J. Marquez, "Robust controller design and PID tuning for multivariable processes," Asian Journal of Control, vol. 4, no. 4, pp. 439-451, 2002. 
[23] B. Vanavil, K. K. Chaitanya, and A. S. Rao, "Improved PID controller design for unstable time delay processes based on direct synthesis method and maximum sensitivity," International Journal of Systems Science, vol. 46, no. 8, pp. 1349-1366, 2015.

[24] J.-P. Richard, "Time-delay systems: an overview of some recent advances and open problems," Automatica, vol. 39, no. 10, pp. 1667-1694, 2003.

[25] P. J. Davis, Interpolation and Approximation, Blaisdell, Boston, Mass, USA, 1975.

[26] O. Boubaker, J. P. Babary, and M. Ksouri, "MIMO sliding mode control of a distributed parameter denitrifying biofilter," Applied Mathematical Modelling, vol. 25, no. 8, pp. 671-682, 2001.

[27] L. Lefèvre, D. Dochain, S. Feyo De Azevedo, and A. Magnus, "Optimal selection of orthogonal polynomials applied to the integration of chemical reactor equations by collocation methods," Computers and Chemical Engineering, vol. 24, no. 12, pp. 2571-2588, 2000.

[28] M. T. Nihtila, T. Damak, and J. P. Babary, "Recursive input delay estimation in linear systems," in Proceedings of the IFAC Symposium on Identification and System Parameter Estimation, July 1994.

[29] Y.-Y. Cao, L. James, and Y.-X. Sun, "Static output feedback stabilization: an ILMI approach," Automatica, vol. 34, no. 12, pp. 1641-1645, 1998.

[30] J. Löfberg, "YALMIP: a toolbox for modeling and optimization in MATLAB," in Proceedings of the IEEE International Symposium on Computer Aided Control Systems Design, pp. 284-289, Taipei, Taiwan, September 2004.

[31] T. Kailath, Linear Systems, Prentice-Hall, New Jersey, NJ, USA, 1980.

[32] A. N. Mete, A. N. Gundes, and H. Özbay, "Decoupled PID controller synthesis for MIMO plants with I/O delays," in Proceedings of the 45th IEEE Conference on Decision and Control (CDC '06), pp. 852-857, San Diego, Calif, USA, December 2006.

[33] B. Friedland, Control System Design: An Introduction to State Space Methods, Dover Publications, New York, NY, USA, 1986.

[34] I.-L. Chien, H.-P. Huang, and J.-C. Yang, "A simple multiloop tuning method for PID controllers with no proportional kick," Industrial and Engineering Chemistry Research, vol. 38, no. 4, pp. 1456-1468, 1999.

[35] K. H. Johansson, "The quadruple-tank process: a multivariable laboratory process with an adjustable zero," IEEE Transactions on Control Systems Technology, vol. 8, no. 3, pp. 456-465, 2000.

[36] F. El Haoussi, E. H. Tissir, F. Tadeo, and A. Hmamed, "Delaydependent stabilisation of systems with time-delayed state and control: application to a quadruple-tank process," International Journal of Systems Science, vol. 42, no. 1, pp. 41-49, 2011. 


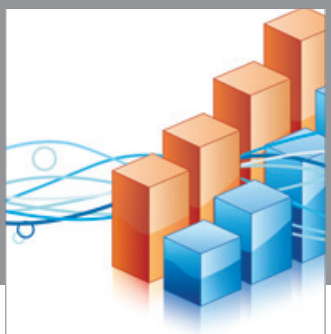

Advances in

Operations Research

vatem alat4

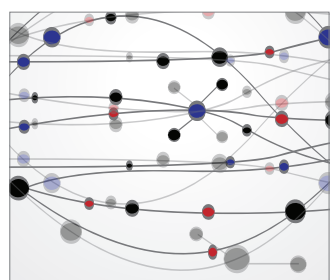

\section{The Scientific} World Journal
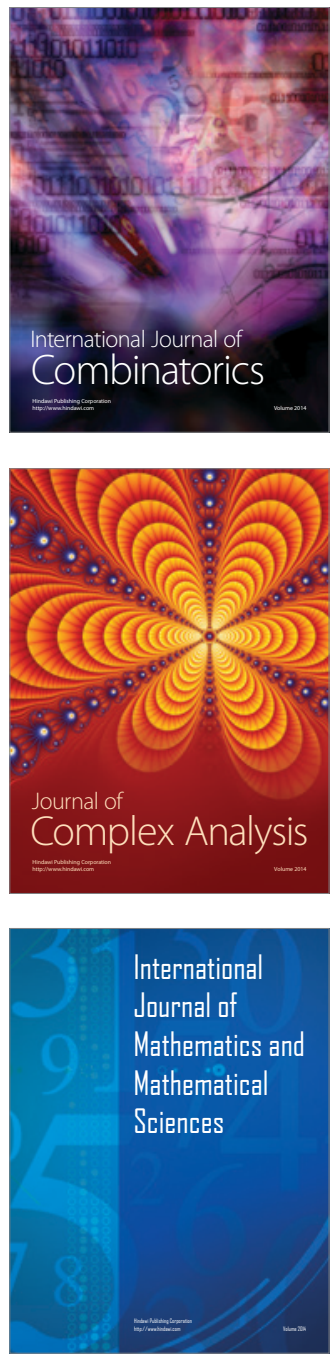
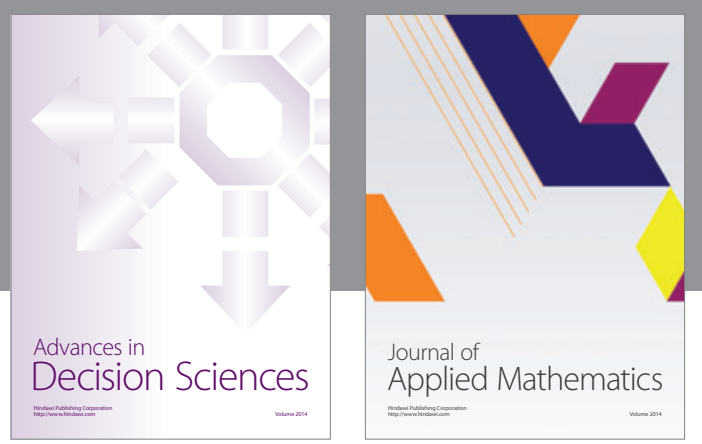

Algebra

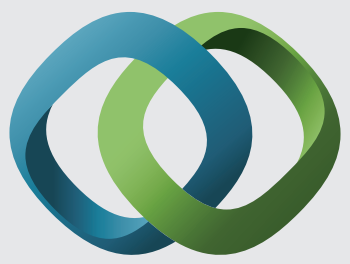

\section{Hindawi}

Submit your manuscripts at

https://www.hindawi.com
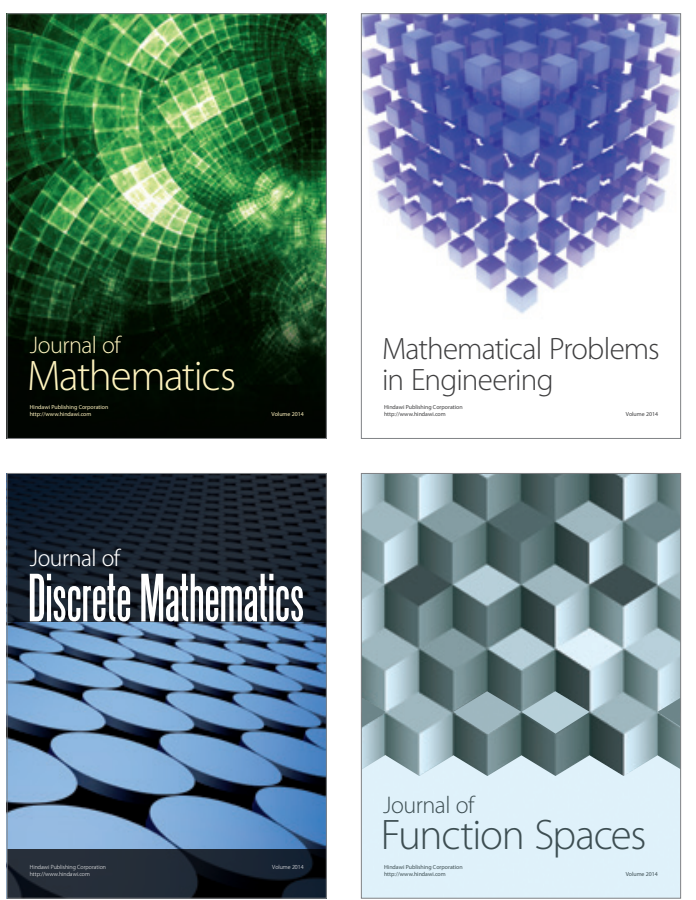

Mathematical Problems in Engineering
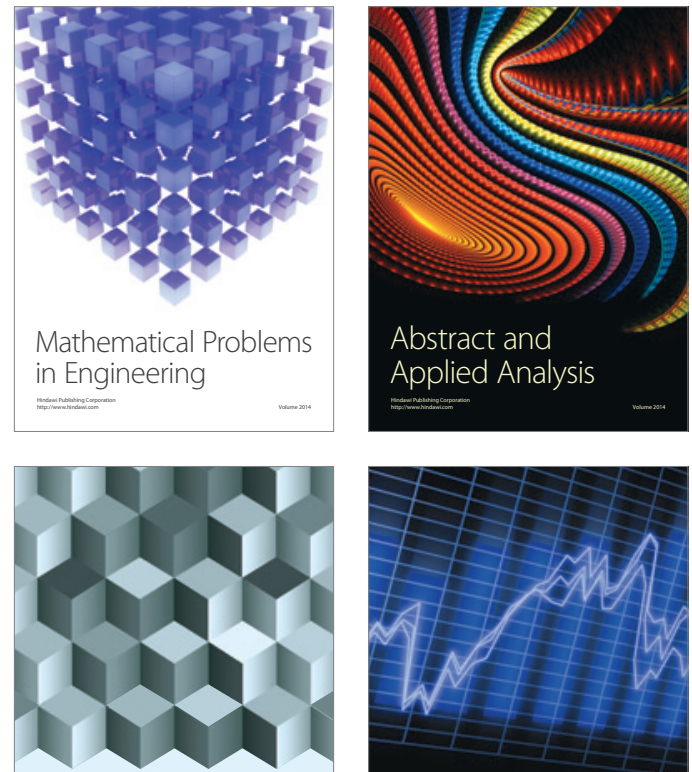

Journal of

Function Spaces

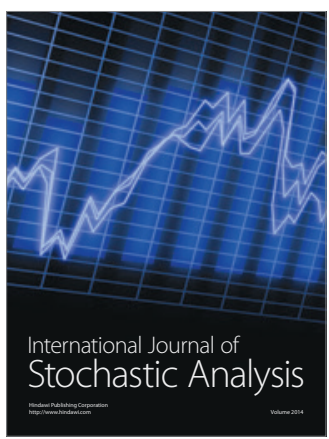

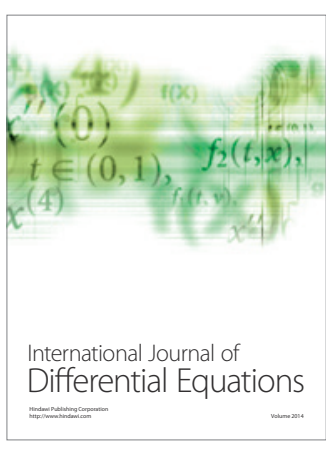
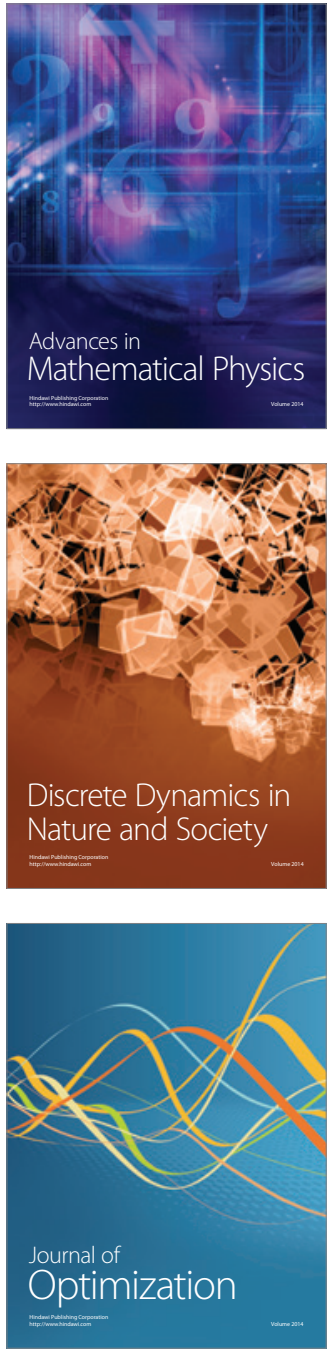